\title{
Deciphering the biosynthetic origin of L-allo-isoleucine
}

Qinglian Li, Xiangjing Qin, Jing Liu, Chun Gui, Bo Wang, Jie Li, and Jianhua Ju*

CAS Key Laboratory of Tropical Marine Bio-resources and Ecology, Guangdong Key Laboratory of Marine Materia Medica, RNAM Center for Marine Microbiology, South China Sea Institute of Oceanology, Chinese Academy of Sciences, Guangzhou 510301, China

*Email: jju@scsio.ac.cn 


\section{Supporting Information}

Table of Contents

Page

S3-S5

S6

S7

S8

S9

S10- S11

S12-S13

S14

$\mathrm{S} 15$

S16

$\mathrm{S} 17$

$\mathrm{S} 18$

S19

S19

S20

S20

S21

S22

S22

S23

S24

S25

S26

23. Figure S18. 'H NMR spectra for the enzymatically produced L-allo-lle and the authentic sample of L-allo-lle.

24. Figure S19. Determination of the equilibrium constant (Keq) for DsaD/DsaE-catalyzed bidirectional reaction.

25. Figure S20. Substrate specificity assays for DsaD/DsaE and MfnO/MfnH.

S27

S28

S29

S29

27. Figure S22. The activity assays of the crossed enzyme pairs DsaD/MfnH and $\mathrm{MfnO} / \mathrm{DsaE}$ using L-Ile or L-allo-lle as the substrate.

28. Figure S23. Activity assays of EcBCAT and MsBCAT from primary metabolism.

S30

S31 $\mathrm{MfnH}$, and DsaE.

30. Figure S25. Effect of PLP supplementation upon the DsaD/MfnO mutant enzyme S32 activities.

31. Figure S26. HPLC profiles of the activity assays of the eight $\mathrm{MfnH}$ mutants as coupled with MfnO using either L-Ile or L-allo-lle as substrates.

32. Figure S27. Phylogenetic tree of DsaE/MfnH with their homologues.
S33-S34

S35 
Table S1. Primers used in this study.

\begin{tabular}{|c|c|c|}
\hline Name & Sequence $\left(5^{\prime}-3^{\prime}\right)$ & Purpose \\
\hline mfnH-DelF & $\begin{array}{l}\text { ATCCGTCGCTACTACGAACTAGTGGACGCGGCGGATTAC } \\
\text { attccggggatccgtcgacc }\end{array}$ & \multirow[t]{2}{*}{ For disrupting $m f n H$} \\
\hline mfnH-DelR & $\begin{array}{l}\text { CTGATCCGCGAAATAGGTGTACCGACGCCAGATCTTCTC } \\
\text { tgtaggctggagctgcttc }\end{array}$ & \\
\hline mfnO-DelF & $\begin{array}{l}\text { ACCTCGGCGTCTTCGGCCGACCGGCGTCGAGAGGCGCT } \\
\text { Cattccggggatccgtcgacc }\end{array}$ & \multirow[t]{2}{*}{$\begin{array}{l}\text { For disrupting } \\
m f n O\end{array}$} \\
\hline mfnO-DelR & $\begin{array}{l}\text { ATACGAATCGGCTGCCTCGCCGCGGTGTACGGCAGCTAG } \\
\text { tgtaggctggagctgcttc }\end{array}$ & \\
\hline mfnH-TF & GAGGAATGGTTCACAGCTGCC & \multirow[t]{2}{*}{$\begin{array}{l}\text { For verifying the } \\
\text { disruption of } m f n H\end{array}$} \\
\hline mfnH-TR & TCTCTTCCAGAGCGATGGCGA & \\
\hline mfnO-TF & ACGACGATCCGATCAGCCGC & \multirow[t]{2}{*}{$\begin{array}{l}\text { For verifying the } \\
\text { disruption of } m f n O\end{array}$} \\
\hline mfnO-TR & CTGTCTGACGGCGCTCCAGT & \\
\hline dsaD-DelF & $\begin{array}{l}\text { GGAGTCGGGCAGTTCGCCGCGCTGGATGCCGAACAGCGC } \\
\text { ACTAGTattccggggatccgtcgacc (Spel site underlined) }\end{array}$ & \multirow[t]{2}{*}{ For disrupting $d s a D$} \\
\hline dsaD-DelR & $\begin{array}{l}\text { ACGGAGCGCTGCGCCGCCCCCGCCTTCGGCACCGCGTTC } \\
\text { ACTAGTtgtaggctggagctgcttc (Spel site underlined) }\end{array}$ & \\
\hline dsaE-DelF & $\begin{array}{l}\text { CAGTGGGGCGAAGAAGTACGTGTCACGCCGACTGAACCG } \\
\text { ACTAGTattccggggatccgtcgacc (Spel site underlined) }\end{array}$ & \multirow[t]{2}{*}{ For disrupting dsaE } \\
\hline dsaE-DelR & $\begin{array}{l}\text { GAGAGCTCTCCCACCGAGGTCAATGAGGCCCGGGTGCGT } \\
\text { ACTAGTtgtaggctggagctgcttc (Spel site underlined) }\end{array}$ & \\
\hline dsaD-TF & GCTGTGACGCGACGAGTT & \multirow[t]{2}{*}{$\begin{array}{l}\text { For verifying the } \\
\text { disruption of } d s a D\end{array}$} \\
\hline dsaD-TR & GGCCGGAGTGCATATCG & \\
\hline dsaE-TF & TCCTGTGGCGGTTCGTG & \multirow{2}{*}{$\begin{array}{l}\text { For verifying the } \\
\text { disruption of } d s a E\end{array}$} \\
\hline dsaE-TR & ACAGCCCGTACACACCG & \\
\hline dsaD-exp-F & TATCATATGGTGCATATCGTGACCAC (Ndel site underlined) & \multirow[t]{2}{*}{$\begin{array}{l}\text { For cloning and } \\
\text { expression of } d s a D\end{array}$} \\
\hline dsaD-exp-R & TATGAATTCTCATGTGATCGCCGAC (EcoRI site underlined) & \\
\hline dsaE-exp-F & TATCATATGATGACCGAGAGCTCTC (Ndel site underlined) & \multirow[t]{2}{*}{$\begin{array}{l}\text { For cloning and } \\
\text { expression of } d s a E\end{array}$} \\
\hline dsaE-exp-R & TATGAATTCTCACACCAGTGGGGCG (EcoRI site underlined) & \\
\hline mfnO-exp-F & AAATTTCCCATATGACCACGACCTCATCCG (Ndel site underlined) & \multirow[t]{2}{*}{$\begin{array}{l}\text { For cloning and } \\
\text { expression of } m f n O\end{array}$} \\
\hline mfnO-exp-R & ATATGAATTCTCAGGCCTCGTGGTTCAC (EcoRI site underlined) & \\
\hline mfnH-exp-F & AAATTTCCCATATGGGGCGCTCCGAGACC (Ndel site underlined) & \multirow[t]{2}{*}{$\begin{array}{l}\text { For cloning and } \\
\text { expression of } m f n H\end{array}$} \\
\hline mfnH-exp-R & ATATGAATTCCTACACCGACTGATCCGC (EcoRI site underlined) & \\
\hline EcBCAT-exp-F & $\begin{array}{l}\text { GATAGGGCGCTAGCATGACCACGAAGAAAGCT } \\
\text { (Nhel site underlined) }\end{array}$ & \multirow{2}{*}{$\begin{array}{l}\text { For cloning and } \\
\text { expression of } \\
\text { EcBCAT }\end{array}$} \\
\hline EcBCAT-exp-R & $\begin{array}{l}\text { CATCGAATTCTTATTATTGATTAACTTGATCTA } \\
\text { (EcoRl site underlined) }\end{array}$ & \\
\hline MsBCAT-exp-F & $\begin{array}{l}\text { GATAGGGCGCTAGCATGAATAGCGGTCCGCT } \\
\text { (Nhel site underlined) }\end{array}$ & \multirow{2}{*}{$\begin{array}{l}\text { For cloning and } \\
\text { expression of } \\
\text { MsBCAT }\end{array}$} \\
\hline MsBCAT-exp-R & $\begin{array}{l}\text { CATCGAATTCTTACTAGTTCAGCCGGGCCATCCA } \\
\text { (EcoRl site underlined) }\end{array}$ & \\
\hline
\end{tabular}




\begin{tabular}{|c|c|c|}
\hline $\begin{array}{l}\text { DsaD-K198L-F } \\
\text { DsaD-K198L-R }\end{array}$ & $\begin{array}{l}\text { GGCACCGGAGCCGCCCTGTGCGCGGGCAA } \\
\text { AGGGCGGCTCCGGTGCCGCCGGCGGCG }\end{array}$ & $\begin{array}{l}\text { For construction of } \\
\text { DsaD/K198L } \\
\text { mutant }\end{array}$ \\
\hline MfnO-K206L-F & GGCACGGGGGCGGCCCTGTGCGGTGGCAA & \multirow{2}{*}{$\begin{array}{l}\text { For construction of } \\
\text { MfnO/K206L } \\
\text { mutant }\end{array}$} \\
\hline MfnO-K206L-R & AGGGCCGCCCCCGTGCCGCCCGGAGCGG & \\
\hline $\begin{array}{l}\text { MfnH-Y10F-F } \\
\text { MfnH-Y10F-R }\end{array}$ & $\begin{array}{l}\text { ACCATCCGTCGCTTTTACGAACTAG } \\
\text { GTAGCGACGGATGGTCTCGGAGCGC }\end{array}$ & $\begin{array}{l}\text { For construction of } \\
\mathrm{MfnH} / \mathrm{Y} 10 \mathrm{~F} \text { mutant }\end{array}$ \\
\hline $\begin{array}{l}\text { MfnH-Y11F-F } \\
\text { MfnH-Y11F-R }\end{array}$ & $\begin{array}{l}\text { ATCCGTCGCTACTTTGAACTAGTGG } \\
\text { AAAGTAGCGACGGATGGTCTCGGAG }\end{array}$ & $\begin{array}{l}\text { For construction of } \\
\mathrm{MfnH} / \mathrm{Y} 11 \mathrm{~F} \text { mutant }\end{array}$ \\
\hline $\begin{array}{l}\text { MfnH-D15L-F } \\
\text { MfnH-D15L-R }\end{array}$ & $\begin{array}{l}\text { TACGAACTAGTG CTG GCGGCGGATT } \\
\text { CAGCACTAGTTCGTAGTAGCGACGG }\end{array}$ & $\begin{array}{l}\text { For construction of } \\
\text { MfnH/D15L mutant }\end{array}$ \\
\hline $\begin{array}{l}\text { MfnH-F26A-F } \\
\text { MfnH-F26A-R }\end{array}$ & $\begin{array}{l}\text { ATGTTCCGTATA GCG TGCGACGACC } \\
\text { CGCTATACGGAACATGGCCTCGTAA }\end{array}$ & $\begin{array}{l}\text { For construction of } \\
\text { MfnH/F26A mutant }\end{array}$ \\
\hline $\begin{array}{l}\text { MfnH-Y32F-F } \\
\text { MfnH-Y32F-R }\end{array}$ & $\begin{array}{l}\text { GACGACCTGATATTTGAGCGGGCCG } \\
\text { AAATATCAGGTCGTCGCAGAATATA }\end{array}$ & $\begin{array}{l}\text { For construction of } \\
\mathrm{MfnH} / \mathrm{Y} 32 \mathrm{~F} \text { mutant }\end{array}$ \\
\hline $\begin{array}{l}\text { MfnH-R34L-F } \\
\text { MfnH-R34L-R }\end{array}$ & $\begin{array}{l}\text { CTGATATACGAGCTGGCCGGAACCG } \\
\text { AGCTCGTATATCAGGTCGTCGCAG }\end{array}$ & $\begin{array}{l}\text { For construction of } \\
\text { MfnH/R34L mutant }\end{array}$ \\
\hline $\begin{array}{l}\text { MfnH-F49A-F } \\
\text { MfnH-F49A-R }\end{array}$ & $\begin{array}{l}\text { GAGTTCCGTCACGCGTATCTCGCCG } \\
\text { CGCGTGACGGAACTCCACGATTCCC }\end{array}$ & $\begin{array}{l}\text { For construction of } \\
\text { MfnH/F49A mutant }\end{array}$ \\
\hline $\begin{array}{l}\text { MfnH-Y50F-F } \\
\text { MfnH-Y50F-R }\end{array}$ & $\begin{array}{l}\text { TTCCGTCACTTCTTTCTCGCCGACC } \\
\text { AAGAAGTGACGGAACTCCACGATT }\end{array}$ & $\begin{array}{l}\text { For construction of } \\
\mathrm{MfnH} / \mathrm{Y} 50 \mathrm{~F} \text { mutant }\end{array}$ \\
\hline $\begin{array}{l}\text { MfnH-R54L-F } \\
\text { MfnH-R54L-R }\end{array}$ & $\begin{array}{l}\text { TATCTCGCCGACCTGAAGATCAGGT } \\
\text { CAGGTCGGCGAGATAGAAGTGACGG }\end{array}$ & $\begin{array}{l}\text { For construction of } \\
\text { MfnH/R54L mutant }\end{array}$ \\
\hline $\begin{array}{l}\text { MfnH-H61F-F } \\
\text { MfnH-H61F-R }\end{array}$ & $\begin{array}{l}\text { AGGTCGGGACGGTTTTCTCTGGACG } \\
\text { AAACCGTCCCGACCTGATCTTGCGG }\end{array}$ & $\begin{array}{l}\text { For construction of } \\
\mathrm{MfnH} / \mathrm{H} 61 \mathrm{~F} \text { mutant }\end{array}$ \\
\hline $\begin{array}{l}\text { MfnH-F79A-F } \\
\text { MfnH-F79A-R }\end{array}$ & $\begin{array}{l}\text { GCCAGAGGAGTCGCGACCGGACAAC } \\
\text { CGCGACTCCTCTGGCGGCGACCCAG }\end{array}$ & $\begin{array}{l}\text { For construction of } \\
\text { MfnH/F79A mutant }\end{array}$ \\
\hline $\begin{array}{l}\text { MfnH-D95L-F } \\
\text { MfnH-D95L-R }\end{array}$ & $\begin{array}{l}\text { ACCCGGTGGGCCCTGTTCCACCAGT } \\
\text { CAGGGCCCACCGGGTGGTCACGGCT }\end{array}$ & $\begin{array}{l}\text { For construction of } \\
\text { MfnH/D95L mutant }\end{array}$ \\
\hline $\begin{array}{l}\text { MfnH-R107L-F } \\
\text { MfnH-R107L-R }\end{array}$ & $\begin{array}{l}\text { AAGATCTGGCGTCTGTACACCTATT } \\
\text { AGACGCCAGATCTTCTCTCCCCGG }\end{array}$ & \multirow{2}{*}{$\begin{array}{l}\text { For construction of } \\
\text { MfnH/R107L } \\
\text { mutant } \\
\text { For construction of }\end{array}$} \\
\hline DsaE-D22L-F & CCGGTTGGTGCTCGCGGACGACGTC & \\
\hline
\end{tabular}




\begin{tabular}{|c|c|c|}
\hline DsaE-D22L-R & AGCACCAACCGGTAGTACTCACGCAC & DsaE/D22L mutant \\
\hline DsaE-D102L-F & GCCTGGAATTCGCCCTCTTCTTTCTGC & \multirow{2}{*}{$\begin{array}{l}\text { For construction of } \\
\text { DsaE/D102L } \\
\text { mutant }\end{array}$} \\
\hline DsaE-D102L-R & AGGGCGAATTCCAGGCGGACTTGGCG & \\
\hline DsaE-H68F-F & GATCGAGAGCGGTCGGTTTACCGTCGCCACGGTCG & \multirow{2}{*}{$\begin{array}{l}\text { For construction of } \\
\text { DsaE/H68F mutant }\end{array}$} \\
\hline DsaE-H68F-R & CGACCGTGGCGACGGTAAACCGACCGCTCTCGATC & \\
\hline
\end{tabular}


Table S2. ${ }^{1} \mathrm{H}$ and ${ }^{13} \mathrm{C}$ NMR spectroscopic data for compound 7 in $\mathrm{CD}_{3} \mathrm{OD}$.

\begin{tabular}{|c|c|c|}
\hline Position & 7 & $\begin{array}{l}7 \\
\delta c\end{array}$ \\
\hline \multicolumn{3}{|l|}{ NMeVal } \\
\hline$\alpha$ & $5.01, d(11.6)$ & 64.0 \\
\hline$\beta$ & $2.34, \mathrm{~m}$ & 26.4 \\
\hline$\Upsilon_{A}-\mathrm{Me}$ & $0.90, d(6.4)$ & 19.8 \\
\hline$\Upsilon_{\mathrm{B}}-\mathrm{Me}$ & $1.11, d(6.4)$ & 20.4, \\
\hline$N M e$ & $3.07, \mathrm{~s}$ & 30.5 \\
\hline$C=O$ & & 169.4 \\
\hline \multicolumn{3}{|l|}{ Leu } \\
\hline$\alpha$ & $5.04, d(7.3)$ & 51.1 \\
\hline$\beta$ & $2.02, \mathrm{~m} ; 1.48, \mathrm{~m}$ & 41.2 \\
\hline$\Upsilon$ & $1.99, \mathrm{~m}$ & 26.3 \\
\hline$\delta_{A}-\mathrm{Me}$ & $0.94, d(6.4)$ & 24.0 \\
\hline$\delta_{\mathrm{B}}-\mathrm{Me}$ & $1.03, d(6.4)$ & 20.0 \\
\hline$C=O$ & & 175.7 \\
\hline \multicolumn{3}{|l|}{ Pip } \\
\hline$\alpha$ & $5.08, \mathrm{~m}$ & 50.3 \\
\hline$\beta$ & $2.77, \mathrm{~d}(15.5) ; 1.94, \mathrm{~m}$ & 32.1 \\
\hline$\Upsilon$ & 3.80 , br s & 62.1 \\
\hline$\delta$ & $2.97, \mathrm{br} \mathrm{s}$ & 54.2 \\
\hline$C=O$ & & 171.7 \\
\hline \multicolumn{3}{|l|}{ D-allo-ile } \\
\hline$\alpha$ & $5.30, d(10.2)$ & 56.6 \\
\hline$\beta$ & $1.87, \mathrm{~m}$ & 36.6 \\
\hline$\beta-\mathrm{Me}$ & $1.10, d(6.1)$ & 16.5 \\
\hline$\Upsilon$ & $1.49, \mathrm{~m} ; 1.24, \mathrm{~m}$ & 27.3 \\
\hline$\delta-\mathrm{Me}$ & $0.98, \mathrm{t}(5.3)$ & 12.2 \\
\hline$C=O$ & & 177.7 \\
\hline \multicolumn{3}{|l|}{ Tyr-OMe } \\
\hline$\alpha$ & $5.10, \mathrm{~m}$ & 54.3 \\
\hline$\beta$ & $3.51, \mathrm{dd}(14.1,4.4)$ & 38.3 \\
\hline 1 & $3.17, \mathrm{~m}$ & 131.5 \\
\hline 2,6 & $7.31, d(8.5)$ & 131.7 \\
\hline 3,5 & $6.77, d(8 . .5)$ & 114.9 \\
\hline 4 & & 160.1 \\
\hline 4-OMe & $3.71, \mathrm{~s}$ & 55.8 \\
\hline$C=O$ & & 174.8 \\
\hline \multicolumn{3}{|l|}{ Thr } \\
\hline$\alpha$ & $4.32, d(3.7)$ & 56.5 \\
\hline$\beta$ & $4.85, \mathrm{~m}$ & 70.3 \\
\hline$\Upsilon-\mathrm{Me}$ & $0.41, d(6.4)$ & 13.7 \\
\hline$C=O$ & & 167.9 \\
\hline \multicolumn{3}{|l|}{$\mathrm{Val}$} \\
\hline$\alpha$ & $4.37, \mathrm{t}(5.0)$ & 58.5 \\
\hline$\beta$ & $2.05, \mathrm{~m}$ & 31.8 \\
\hline $\mathrm{r}_{\mathrm{A}}-\mathrm{Me}$ & $0.97, d(6.4)$ & 19.0 \\
\hline$\Upsilon_{\mathrm{B}}-\mathrm{Me}$ & $0.87, d(6.4)$ & 18.3 \\
\hline$H C=O$ & $8.12, \mathrm{~s}$ & 164.0 \\
\hline$C=O$ & & 172.5 \\
\hline
\end{tabular}


Table S3. Crystal data and structure refinement for compound 7. (03162A)

\begin{tabular}{|c|c|c|}
\hline Identification code & \multicolumn{2}{|l|}{ 03162A } \\
\hline Empirical formula & \multicolumn{2}{|l|}{ C86 H162 N16 O35 } \\
\hline Formula weight & \multicolumn{2}{|l|}{1980.31} \\
\hline Temperature & \multicolumn{2}{|l|}{$150(2) \mathrm{K}$} \\
\hline Wavelength & \multicolumn{2}{|l|}{$1.54184 \AA$} \\
\hline Crystal system & \multicolumn{2}{|l|}{ Orthorhombic } \\
\hline Space group & \multicolumn{2}{|l|}{$\mathrm{P} 22_{1} 2$} \\
\hline \multirow[t]{3}{*}{ Unit cell dimensions } & $\mathrm{a}=24.9455(2) \AA$ & $\alpha=90^{\circ}$ \\
\hline & $\mathrm{b}=19.28742(18) \AA$ & $\beta=90^{\circ}$. \\
\hline & $\mathrm{c}=11.27639(9) \AA$ & $\gamma=90^{\circ}$ \\
\hline Volume & \multicolumn{2}{|l|}{$5425.46(9) \AA^{3}$} \\
\hline $\mathrm{Z}$ & \multicolumn{2}{|l|}{2} \\
\hline Density (calculated) & \multicolumn{2}{|l|}{$1.212 \mathrm{Mg} / \mathrm{m}^{3}$} \\
\hline Absorption coefficient & \multicolumn{2}{|l|}{$0.783 \mathrm{~mm}^{-1}$} \\
\hline $\mathrm{F}(000)$ & \multicolumn{2}{|l|}{2140} \\
\hline Crystal size & \multicolumn{2}{|c|}{$0.420 \times 0.390 \times 0.340 \mathrm{~mm}^{3}$} \\
\hline Theta range for data collection & \multicolumn{2}{|l|}{2.896 to $67.037^{\circ}$. } \\
\hline Index ranges & \multicolumn{2}{|c|}{$-29<=\mathrm{h}<=29,-23<=\mathrm{k}<=20,-13<=\mathrm{l}<=13$} \\
\hline Reflections collected & \multicolumn{2}{|l|}{47323} \\
\hline Independent reflections & \multicolumn{2}{|c|}{$9610[\mathrm{R}(\mathrm{int})=0.0319]$} \\
\hline Completeness to theta $=67.037^{\circ}$ & \multicolumn{2}{|l|}{$99.6 \%$} \\
\hline Absorption correction & \multicolumn{2}{|c|}{ Semi-empirical from equivalents } \\
\hline Max. and min. transmission & \multicolumn{2}{|l|}{1.00000 and 0.71465} \\
\hline Refinement method & \multicolumn{2}{|c|}{ Full-matrix least-squares on $\mathrm{F}^{2}$} \\
\hline Data / restraints / parameters & \multicolumn{2}{|l|}{$9610 / 0 / 635$} \\
\hline Goodness-of-fit on $\mathrm{F}^{2}$ & \multicolumn{2}{|l|}{1.054} \\
\hline Final $\mathrm{R}$ indices $[\mathrm{I}>2 \operatorname{sigma}(\mathrm{I})]$ & \multicolumn{2}{|c|}{$\mathrm{R} 1=0.0457, \mathrm{wR} 2=0.1275$} \\
\hline $\mathrm{R}$ indices (all data) & \multicolumn{2}{|c|}{$\mathrm{R} 1=0.0472, \mathrm{wR} 2=0.1293$} \\
\hline Absolute structure parameter & \multicolumn{2}{|l|}{$-0.03(4)$} \\
\hline Extinction coefficient & \multicolumn{2}{|l|}{$\mathrm{n} / \mathrm{a}$} \\
\hline Largest diff. peak and hole & \multicolumn{2}{|l|}{1.156 and -0.388 e. $\AA^{-3}$} \\
\hline
\end{tabular}


A

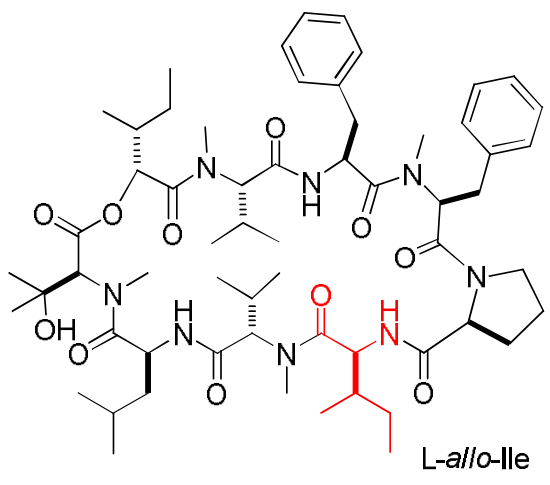

aureobasidin A

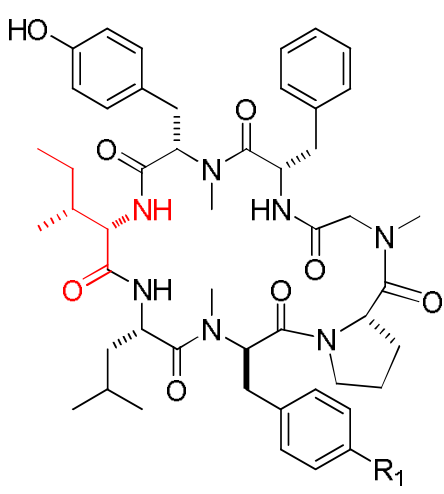

cordyheptapeptides $1 \mathrm{R}_{1}=\mathrm{H}$ $2 \mathrm{R}_{1}=\mathrm{OH}$

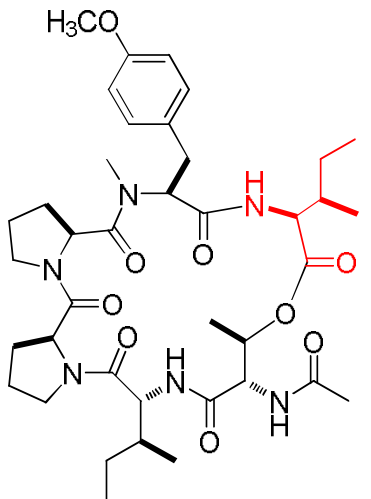

aspergillicin $\mathrm{E}$

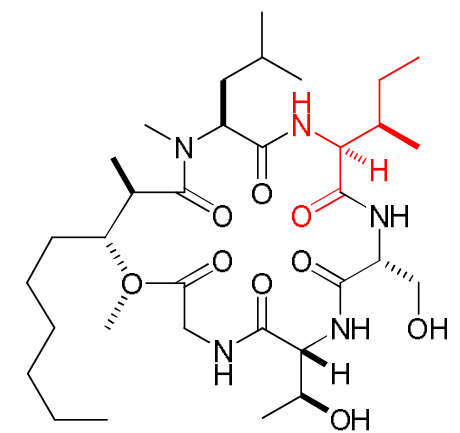

globomycin

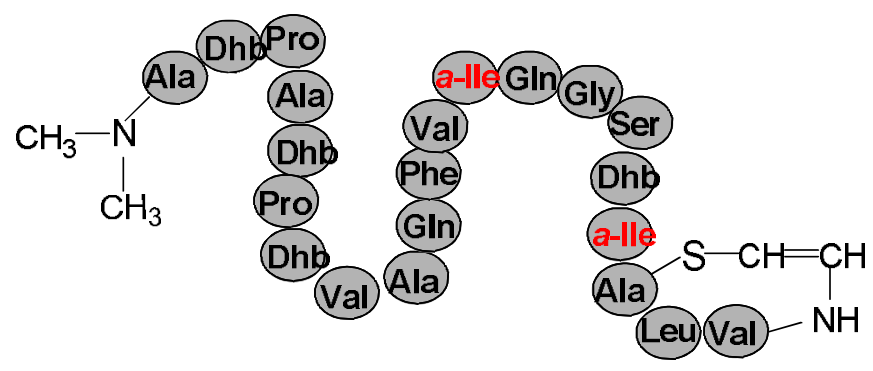

cypemycin

B
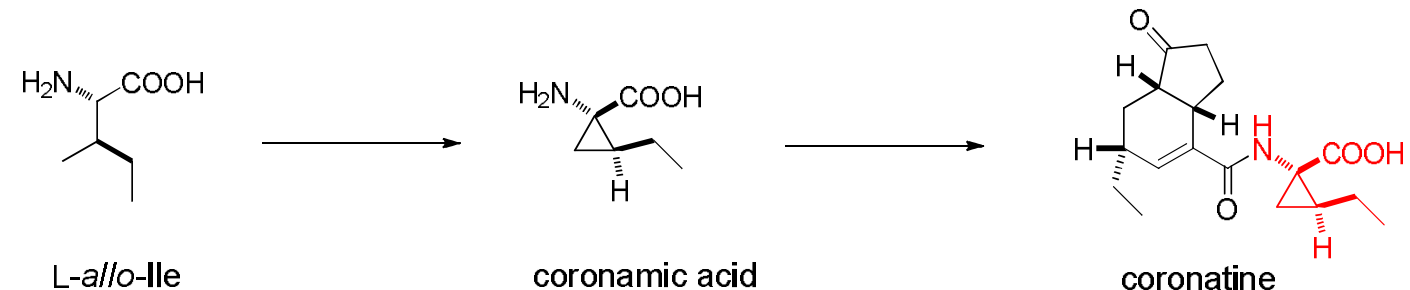

Figure S1. (A) Selected natural products containing the L-allo-lle moiety (colored red). (B) Lallo-lle is used as a precursor to synthesize phytotoxin coronatine. 

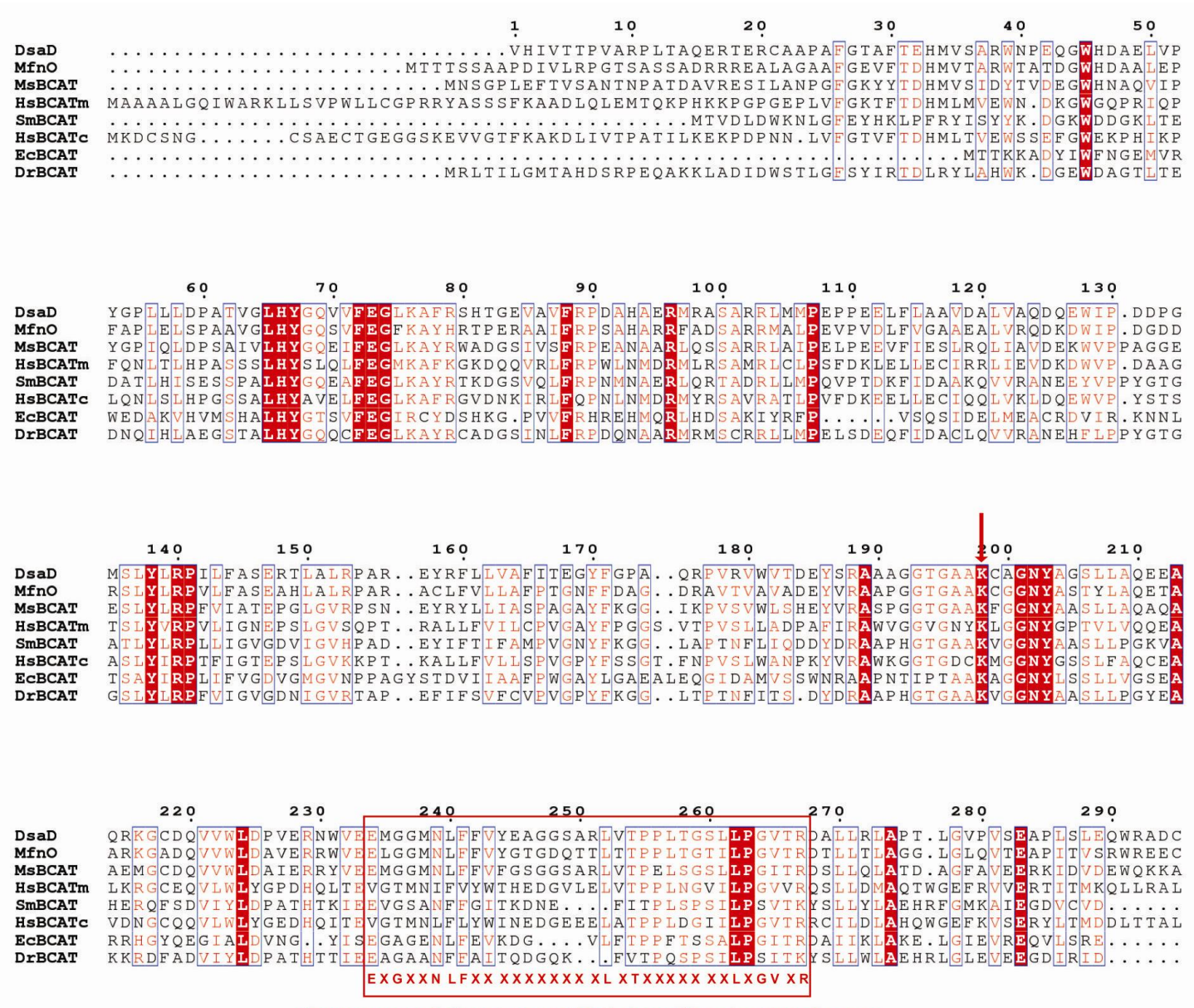

the conserved signature motif of class-IV aminotransferases

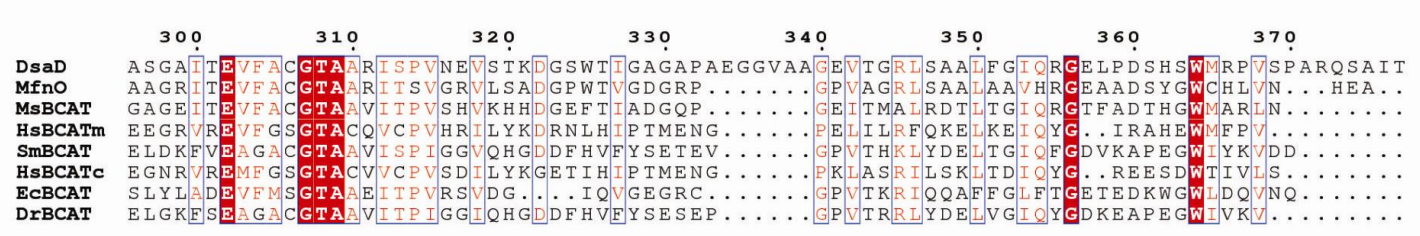

Figure S2. Sequence alignments of $\mathrm{DsaD} / \mathrm{MfnO}$ with the branched-chain aminotransferases (BCATs) from Mycobacterium smegmatis (MsBCAT, NCBI gi number: 399233140), human mitochondrial (hsBCATm, NCBI gi number: 13786631), Streptococcus mutans (SmBCAT, NCBI gi number: 436408788), human cytosolic (hsBCATc, NCBI gi number: 75766442), E.coli (EcBCAT, NCBI gi number: 30749295), and Deinococcus radiodurans (DrBCAT, NCBI gi number: 429544222). The conserved EXGXXNLFX ${ }_{n} L X T X_{n} L X G V X R$ signature motif is indicated by the red box. The PLP binding active-site lysine is indicated with a vertical arrow. 
MlnH

DsaE

W 030868916. 1

W 013496225. 1

W_014051297. 1

WP 009886289. 1

W_021786773. 1

W 029211959. 1

WP 006946988. 1

WP 038566685. 1

W_035303624. 1

$\mathrm{KSI}_{\mathrm{ct}}$

$\mathrm{MnH}$

DsaE

WP 030868916. 1

WP 013496225. 1

W 014051297. 1

WP_009886289. 1

WP 021786773. 1

W_029211959. 1

W_006946988. 1

WP 038566685. 1

W 035303624. 1

$\mathrm{KSI}_{\mathrm{ct}}$

MinH

DsaE

W 030868916. 1

WP 013496225. 1

W_ 014051297. 1

W 009886289. 1

W 021786773. 1

W_029211959. 1

W 006946988. 1

W 038566685. 1

W_ 035303624. 1

$\mathrm{KSI}_{\mathrm{ct}}$
MinH Tyr 10/ KSI ct-Tyr14 MinH Arg34/ KSI ct-Asp38 - - - MR - - - SEII RRYYELVAADYEAMFR FODDU YERA GIEPI EG MESSPIEYNEARMEYRLVADDMLLVSLFAEDAYRRP- GEPIRG - MEASR - - ESTVREYYERVDADYEAYFDMFCEDVERG GIEAI VG MADDL - - - RSKAYLYYQRVDAGD EG IALFFPEWYERG GPPI HG - - - MEP- - - - AQVARRYYELVDEEAYEELVG FIKDVTYERP- GESI DG

- - MEDL - - - KDIKKYYI AVDKNDDIL FSI FADN YKRP- GEPI EG

- - MEN - - - EKLWKYYAAVDAKDDIL FSVFSDN YYKRP- GEPI EG MTTAP - - - - APVTRYELVDANDDQLVALFIPDATYERP- GEPMRG MQRESR - - - DAH RRYYWDAGDEGMD FIDDATYQRP- GYPMAG MQRESG - - - DAH RRYYEWDAGDEGMD FIDDATYCRP- GYPMAG MNDD - . - - - WLHYELVANDPAL LALFADDAYHRP- GDPLVG MNIPEH - - MAWQRYAALNAGD DO VALFADDATVEDPGSEPRSG

I VEFRFFYADRKI RSGRSLDM ENGDWAARGMTCQRRIGAVITR HIGTAFYTGERM ESCRHIVATWARGDVAVMGEGLLDORQRLE MEFKRFYLADR I ESCRHDASI VESGWVAARGYFTCQLKTAEEVSVN LDALRQFI GER VRECRFL FRMVECHNAVRGRFGMLKSAPMDR RAV RQFIDRDRP SNEFFLDSWSDOA VAVRGIFRGQ DOHQELG MANFKEFYQKNR I KECHILSN I ASDPYM VEGEFNG LKDOSKSHTT MEKFREFYRGNM RECHILSN I KKEPYWEGFFNG LKDOSKSHT HSGTAFYSGER I ASGAHLTHIWDODEVAVQRRAGTARDORLDR REALRAFYSGDR ESCRSVISL U DODEAFVRGFNOMLDOSDAHE REALRAFYSGRM ESCRHSVIS U DODEAFVRGFFNGLLDOSDAHE QDMRFYQDERM AACH HDQLVACD EI AVGIFTGILRDSID TLR TAAI REFYANSLK PLAVELTQEVRAVANEAAFAFTISFEYQ GKTWA

\section{MinH Asp95/ KSI ct Asp99}

WADFHQR GEKI WRRYTYFADOSV - - -

FADFFLLNERRESRRDIYFFAPLV - - -

WADFHFR GOKI WRRYIYFADRAI - - - -

FADFHFR DA I WRRYSYFMREV- - - -

FADFFEFE DOKI ARRYIFTDRDIV - . -

FVMYIFS SCKAI KRHIYFDOOSV - - -

FVDYIFS- NCKAYKRHIFDOQN- - - -

FADFFR DOER I AYRITYFYAPLA - - -

FADFFRFGAGRI AYRQIYFYAPLV - - -

FADFFRFGAGH AYRQIYFYAPLV - - -

FADFFR NRIG I YRRDIFFFAPLV - - -

PI DHRENGAGWSMRALFEKN HACA

Figure S3A. Alignment of amino acid sequences of DsaE/MfnH and their homologous proteins

with delta -3 -ketosteroid isomerase from Comamonas testosteroni $\left(\mathrm{KSI}_{\mathrm{ct}}\right)$. The following 
proteins (with GeneBank IDs) were used for amino acid alignment: WP_030868916.1 from Streptomyces sp. NRRL F-2747; WP_013496225.1 from Thermaerobacter marianensis; WP_014051297.1 from Halophilic archaeon DL31; WP_009886289.1 from Ferroplasma acidarmanus; WP_021786773.1 from Ferroplasma sp. Type II; WP_029211959.1 from Arsenicicoccus bolidensis; WP_006946988.1 from Dermacoccus sp. Ellin185; WP_038566685.1 from Dermacoccus nishinomiyaensis; WP_035303624.1 from Actinokineospora inagensis. The amino acids in DsaE/MfnH and their homologous proteins corresponding to the three catalytic amino acids of $\mathrm{KSI}_{\mathrm{ct}}$ (Tyr14, Asp38 and Asp99) are shaded in gray. 
MinH
DsaE
W_029211959. 1
W_006946988. 1
W_038566685. 1
W_035303624. 1
W_030868916. 1
W_013496225. 1
W_012855066. 1
W_014051297. 1
W_009886289. 1
W_021786773. 1

$\mathrm{MlnH}$

DsaE

W 029211959. 1

WP_006946988. 1

W_038566685. 1

W'035303624. 1

W_030868916. 1

W_013496225. 1

WP 012855066. 1

W_014051297. 1

W_009886289. 1

W_021786773. 1

$\mathrm{MnH}$

DsaE

WP 029211959. 1

W_006946988. 1

WP 038566685. 1

WP 035303624. 1

WP 030868916. 1

W_013496225. 1

W-012855066. 1

WP_014051297. 1

WP_009886289. 1

W_021786773. 1
MnH Asp15/ DsaE-Asp22 M MnH Arg34/ DsaE Arg41

- - - - MQR - - - SEII RRYYELDAADYEAMFR FODU YERAGIEPI EG VEFRFYL

- - MESSPIEVNEARMEYYRLVADDLCLVSI FAEDAVYRRPGYPIRGITGTAFYT

- - MTTAP- - - - APVIRYYELVANDDCLVAI FIPDATYERPGYEPMRGBGTAFYS

- - NQRESR - - - DAH RRYYWDAGMEGMD FIDDATYQRPGEPMACREALRAFYS

- - MQRESG - - - DAH RRYEWDAGDEGMDI FIDDATYRPGEPMACREALRAFY

- - MNID - - - - - WLHYELVDANDPALLAL FADDAAYHPGIPLVGQDMRFYQ

- - - MEASR - - - ESTVREYYERMAADYEAVFDMFCEDLYERCGIEAI VGMEFKRFYL

- - MADDL - - - RSKAYLYYQRDAGD EG LAL FHEWYERCCRPPI HADALRQFY

MAHATTIL - - - ESI VRSYEHDAHR EEMAI FDEN VYERQGTPII VG EAL RRFYE

- - - - - MEP- - - - AQVARRYYELVDEAYEELVG FIKDVIYERPCQESI DQRAV RQFYD

- - MED KD - - - - TYKKYYI AVDKD DIL FSI FADN VYKRPGYPI EGMANFKEFYQ

- - MENEK - - - - LKKYYAAMAKDDIL FSVFSDN VYKRPGEPI EGMEKFREFYR

MnH H s61/ DsaE H s68

MinH Asp95/ DsaE- Asp102 MnH Arg107/ DsaE Arg115

ADRK RSCRISLDM ENGDWAARGMTCQRIGEAVITRMADFHER GEK WRYTY

GER ESGRIIVATWARGOVAVNGEGMKDORQMLEFADFFLLNERRFSR DIY

GER I ASGAFILTHWDOAEVAVQREAGTARDORMDLREDFFR DOER I AYRITY

GDRM ESCRISVISL I DODFAFVRGFNGL DOSDDAHEFADFFRFGAGR AYRQIY

GRR ESCRISVISLU DOEFAFVRGFNGL KDOSDAH LFFADFFRFGAGH AYRQIY

DERM AAGH HDQLVAGDEI AVGIFTGILRDSID TLRFADFFR NRIG YRRDIF

ADR I ESCRHDASI VESGDWAARGMTGQLKAEEVSVNADFH LR GOK WRYIY

GHR VRERR EI FRMLECPHAVRRFRGL KSGEPMVRFADFH HR DA I WRYSY

HER I ESCR FL DQM PGIDWAVRGFECRLROAQRLRFTDM FR DOR VRESL

RDRPL SNEE EI DSWSDOA VAVGGIFREQ DOHQELFADF EFE- DOK AR YIF

KNR I KECHIL SN I ASDPYM VEGFFNA LKOSKSHITFVDYTFS- SGKAI KRHY

GRY RECHIL SN I VKEPYWECFFNG LKDOSKSHITFVDYIFS- NGKAKKRHY

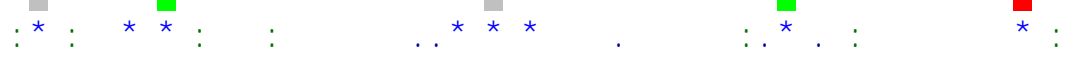

FADOSV

FFAPLV

FYAPLA

FYAPLV

FYAPLV

FFAPLV

FADRAI

FMDRFV

FPDEA

TDRDIV

FDOSS

FDOQN

Figure S3B. Alignment of amino acid sequences of DsaE/MfnH with their homologous proteins of unknown function by Clustal W (1.81). Ten hits of the BLAST search of MfnH from the NCBI 
database were aligned: WP_029211959.1 from Arsenicicoccus bolidensis; WP_006946988.1 from Dermacoccus sp. Ellin185; WP_038566685.1 from Dermacoccus nishinomiyaensis; WP_035303624.1 from Actinokineospora inagensis; WP_030868916.1 from Streptomyces sp. NRRL F-2747; WP_013496225.1 from Thermaerobacter marianensis; WP_012855066.1 from Thermomonospora curvata; WP_014051297.1 from Halophilic archaeon DL31; WP_009886289.1 from Ferroplasma acidarmanus; WP_021786773.1 from Ferroplasma sp. Type II. The fully conserved residues in $\mathrm{DsaE} / \mathrm{MfnH}$ and their homologues are indicated by blue asterisks. The eight conserved polar amino acids of DsaE/MfnH were demonstrated to have no effect on the enzymatic activity as determined by mutagenesis studies; these are shaded in gray. The four conserved polar amino acids of DsaE/MfnH whose mutation was found to sharply diminish enzymatic activity are shaded in green; and the catalytic arginine residues of DsaE/MfnH are shaded in red. 


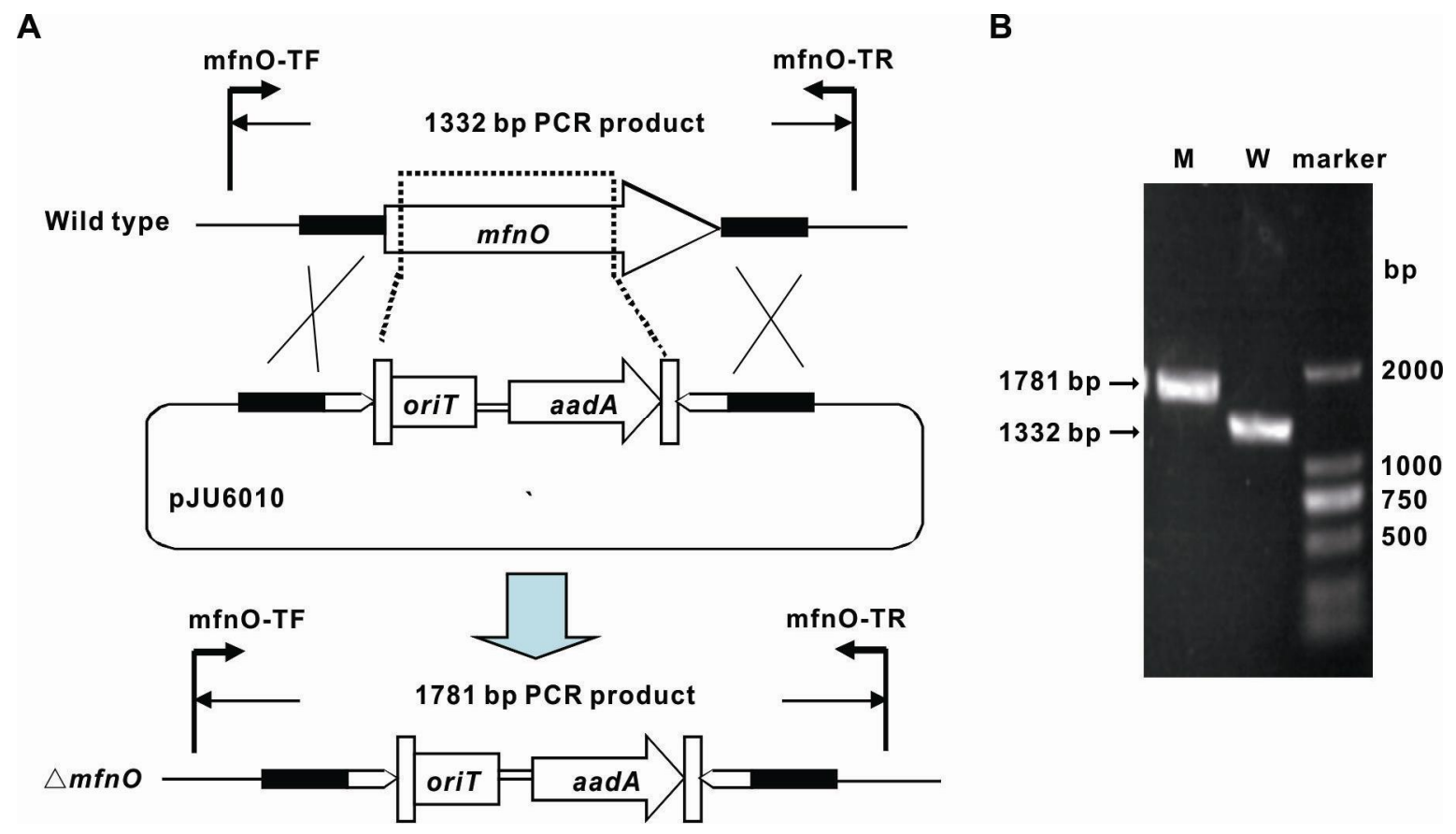

Figure S4. Disruption of $m f n O$ in wild-type $S$. drozdowiczii SCSIO 10141 via PCR-targeting. (A) Schematic representation for disruption of $m f n O$. (B) PCR analyses of the wild-type strain and the mfnO double-cross mutant carried out using the primers listed in Table S1. Marker: DNA molecular ladder; W: using the genomic DNA of S.drozdowiczii SCSIO 10141 as template; M: using the genomic DNA of $m f n O$ mutant as template. 
A

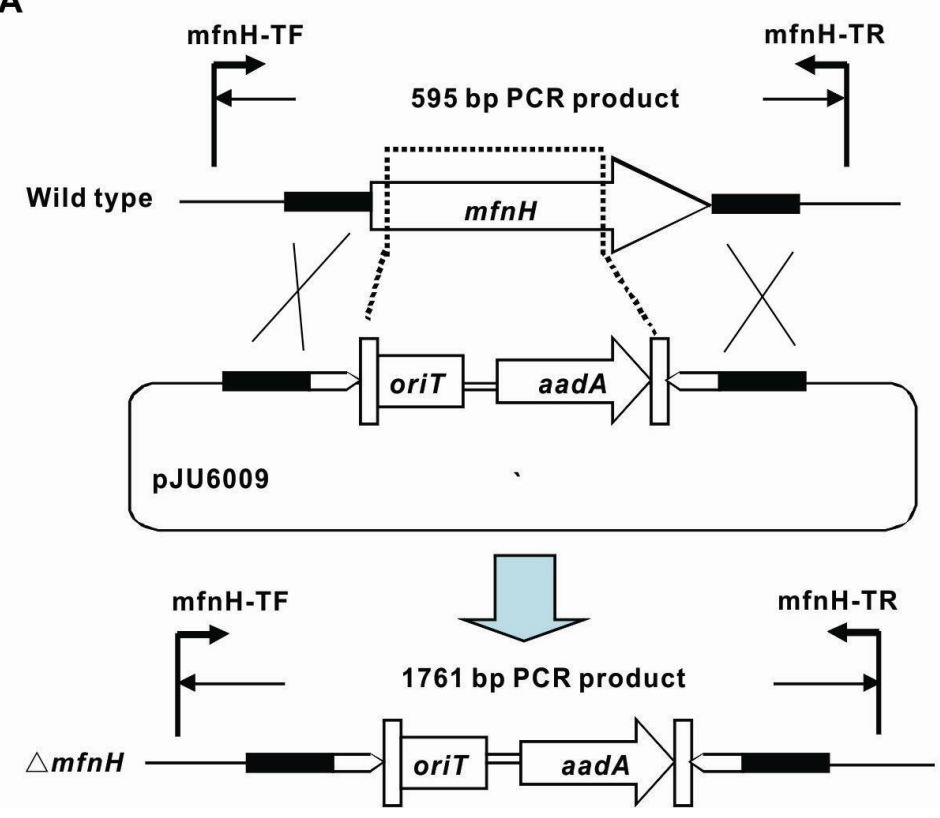

B

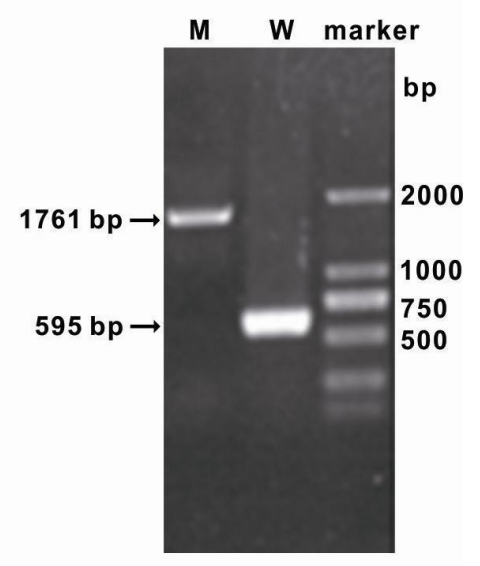

Figure S5. Disruption of $m f n H$ in wild-type $S$. drozdowiczii SCSIO 10141 via PCR-targeting. (A) Schematic representation for disruption of $m f n H$. (B) PCR analyses of the wild-type strain and the $m f n H$ double-cross mutant carried out using the primers listed in Table S1. Marker: DNA molecular ladder; W: using the genomic DNA of S. drozdowiczii SCSIO 10141 as template; M: using the genomic DNA of $\mathrm{mfnH}$ mutant as template. 


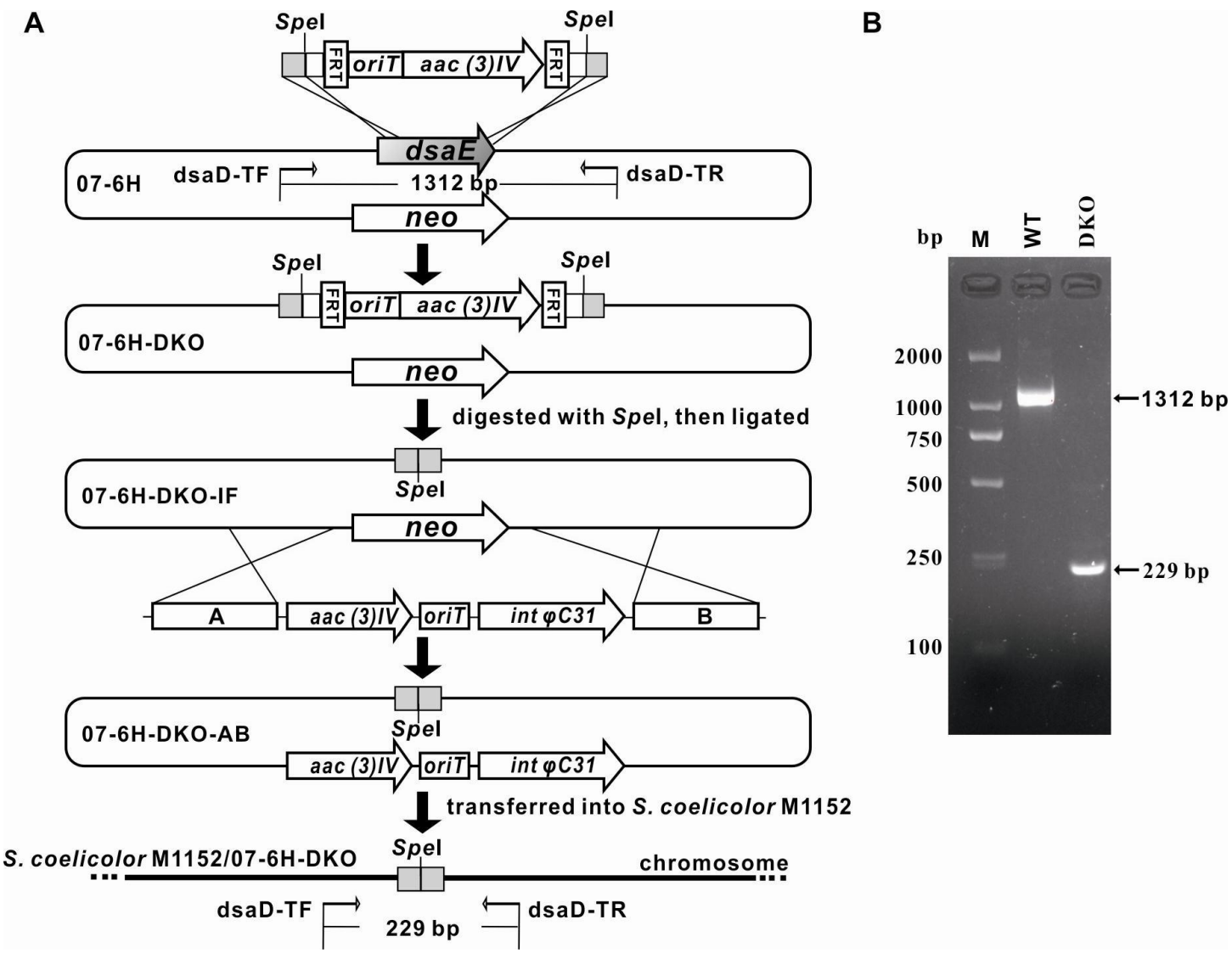

Figure S6. In-frame disruption of $d s a D$ within the DSA gene cluster and subsequent heterologous expression in S. coelicolor M1152. (A) Schematic representation for disruption of dsaD. (B) PCR analysis of $S$. coelicolor M1152/07-6H-DKO carried out using the primers listed in Table S1. M: DNA molecular ladder; WT: using cosmid 07-6H as template; DKO: using the genomic DNA of S. coelicolor M1152/7-6H-DKO as template. 


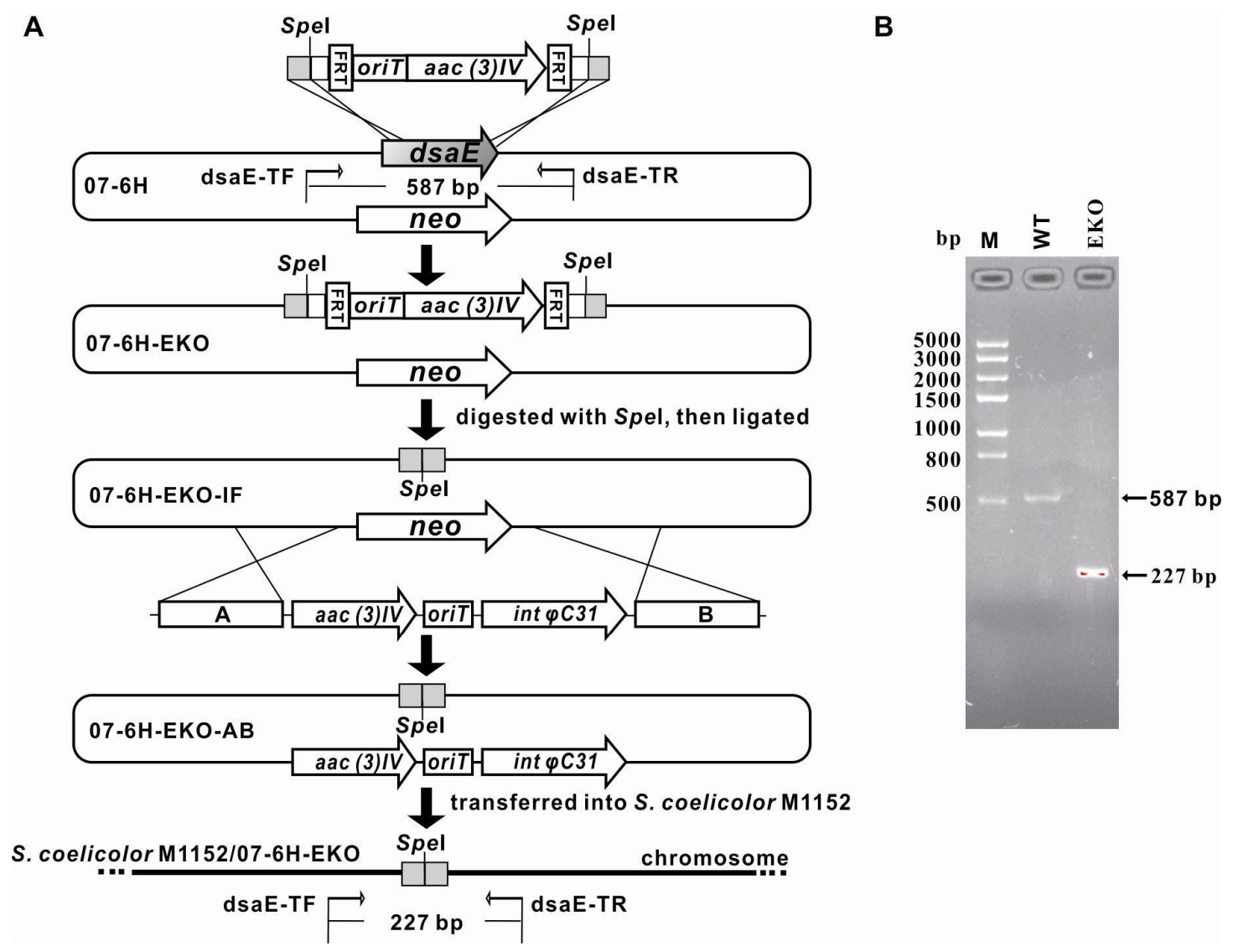

Figure S7. In-frame disruption of dsaE within the DSA gene cluster and subsequent heterologous expression in S. coelicolor M1152. (A) Schematic representation for disruption of dsaE. (B) PCR analysis of $S$. coelicolor M1152/07-6H-EKO carried out using the primers listed in Table S1. M: DNA molecular ladder; WT: using cosmid 07-6H as template; EKO: using the genomic DNA of S. coelicolor M1152/7-6H-EKO as template. 


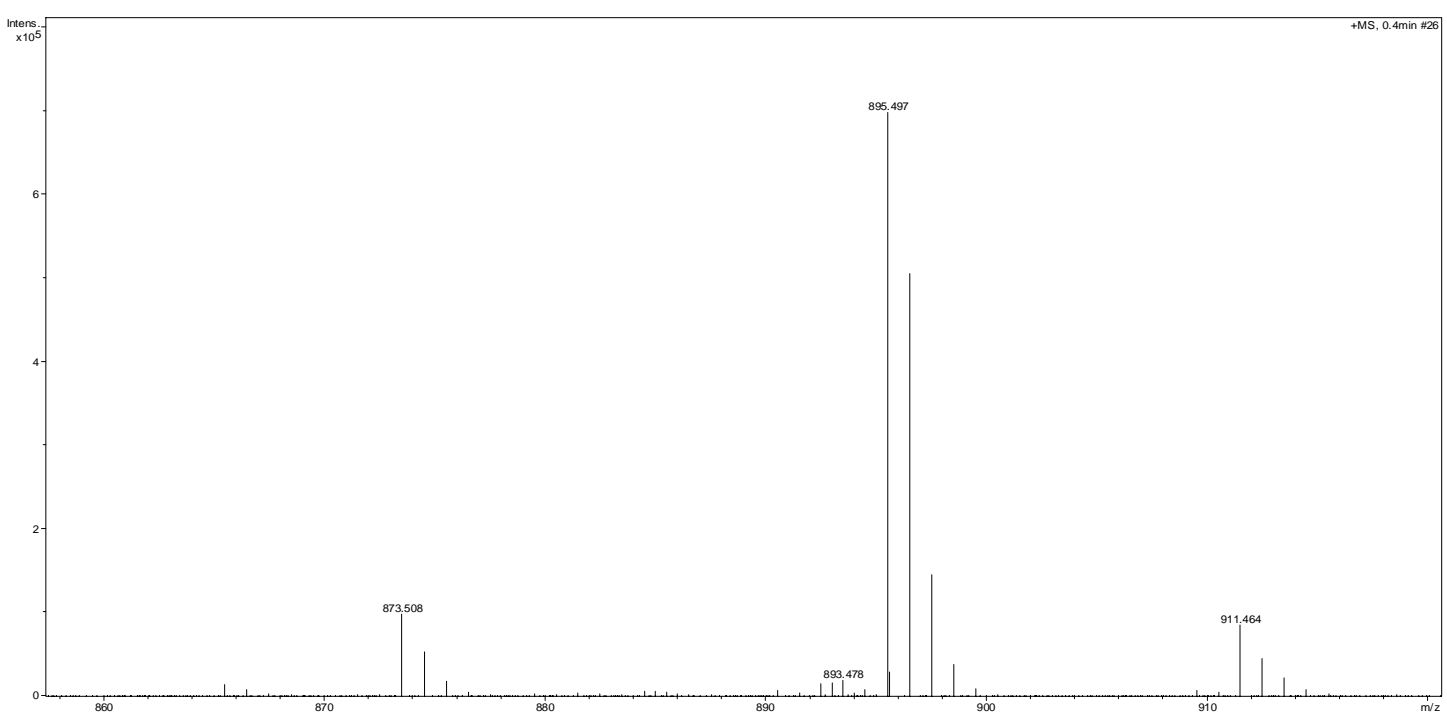

Figure S8. HRESIMS spectrum of compound 7. 


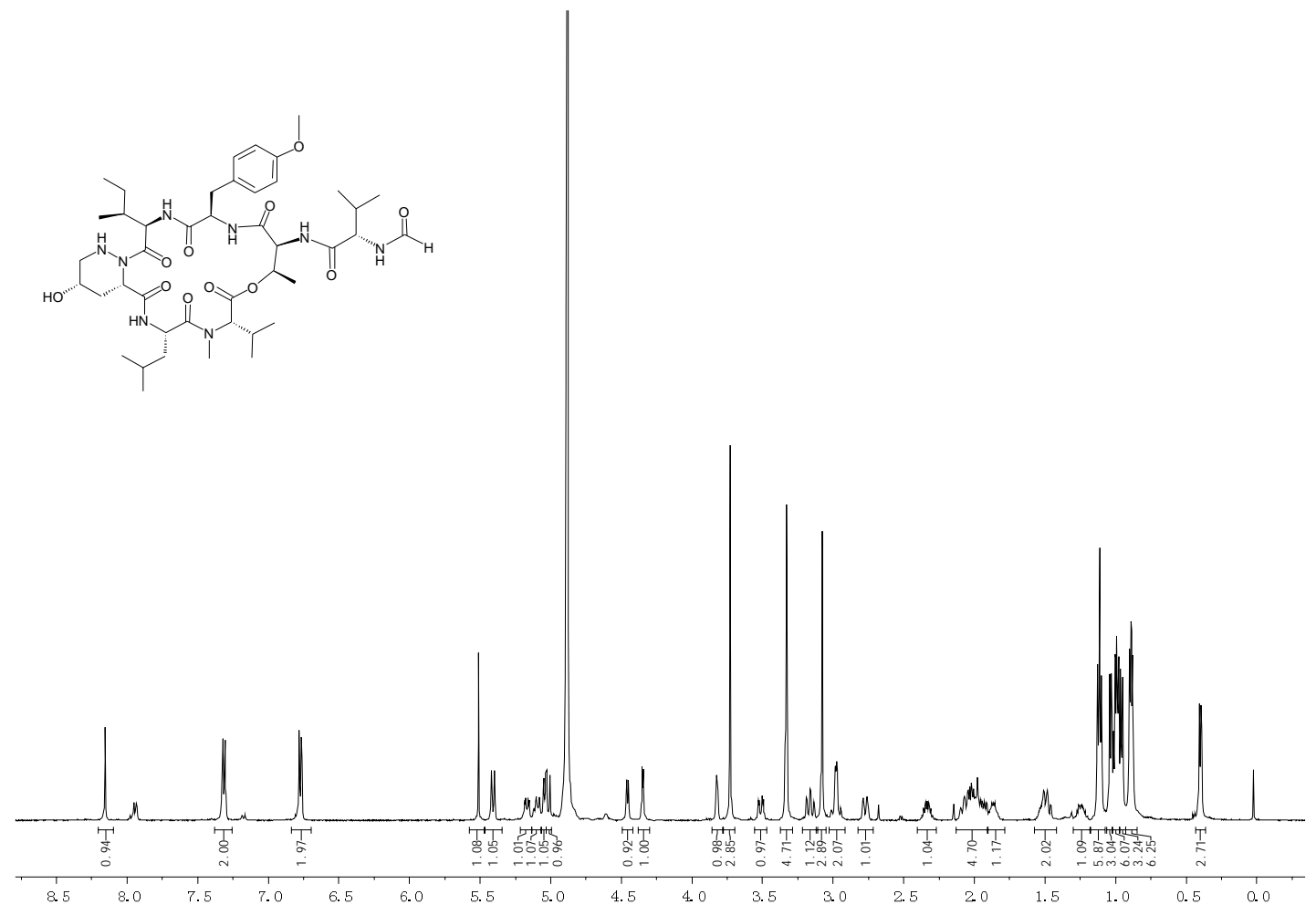

Figure S9. ${ }^{1} \mathrm{H}(500 \mathrm{MHz}) \mathrm{NMR}$ spectrum of compound 7 in MeOD.
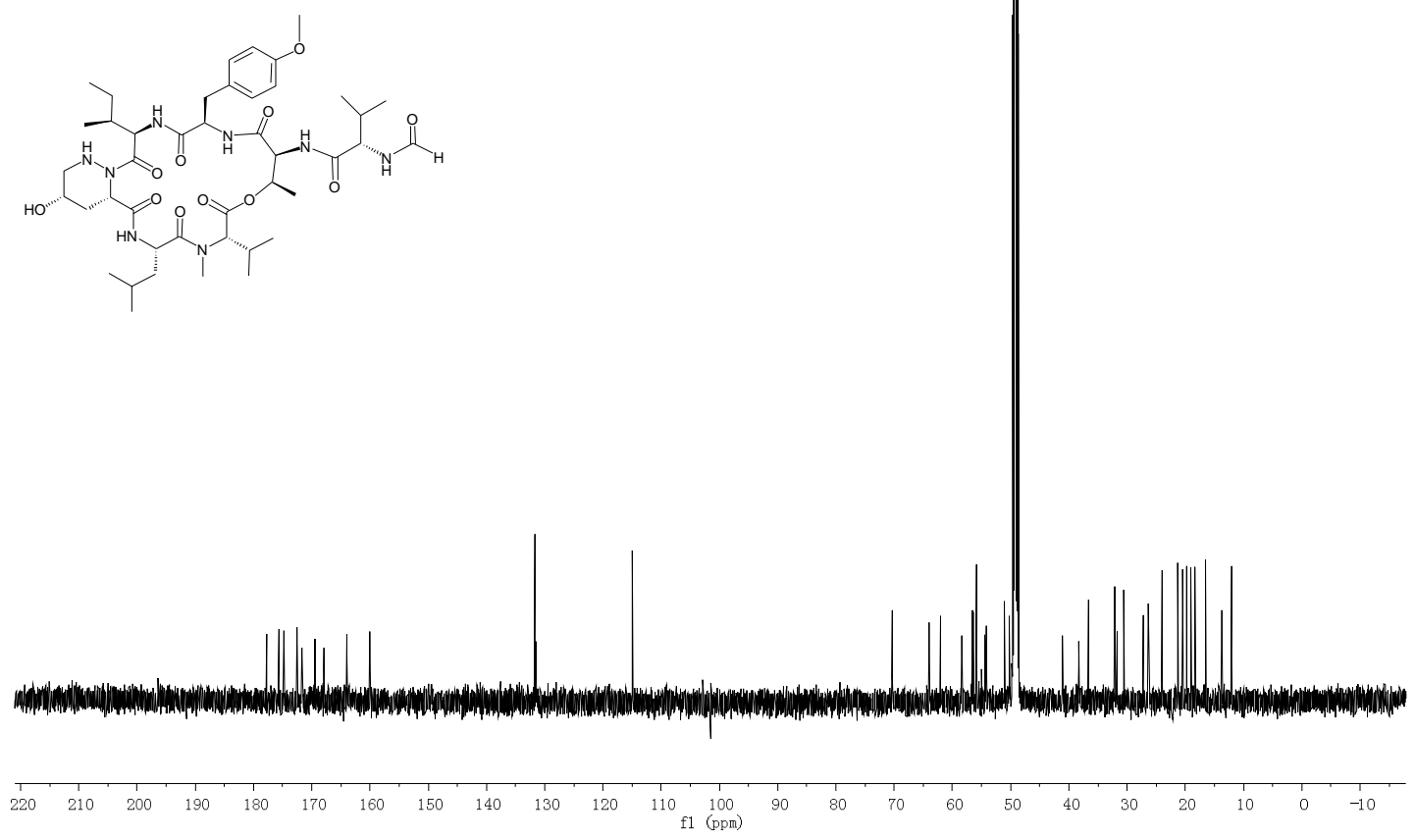

Figure S10. ${ }^{13} \mathrm{C}(500 \mathrm{MHz})$ NMR spectrum of compound $\mathbf{7}$ in MeOD. 


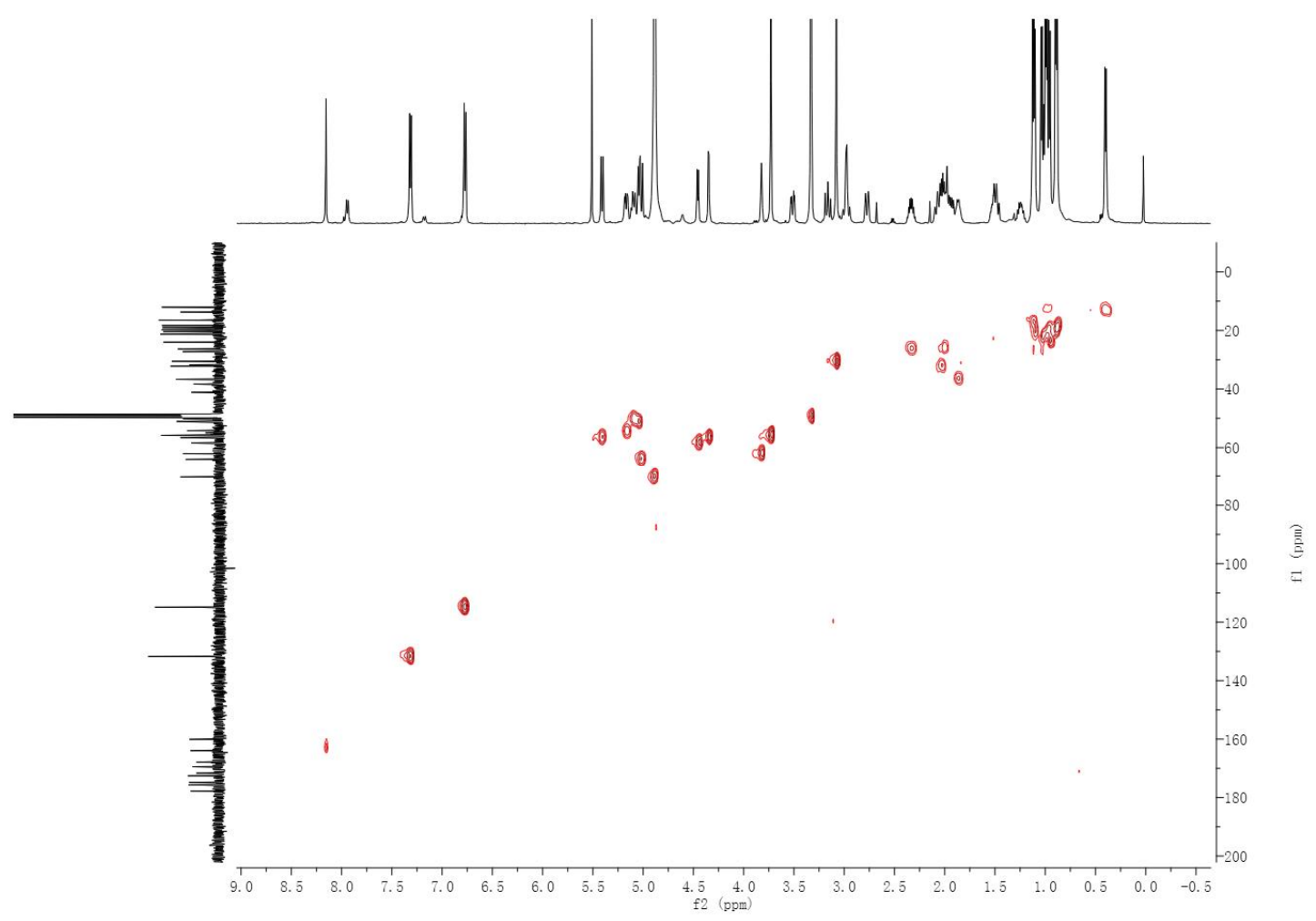

Figure S11. HSQC NMR spectrum of compound 7 in MeOD.

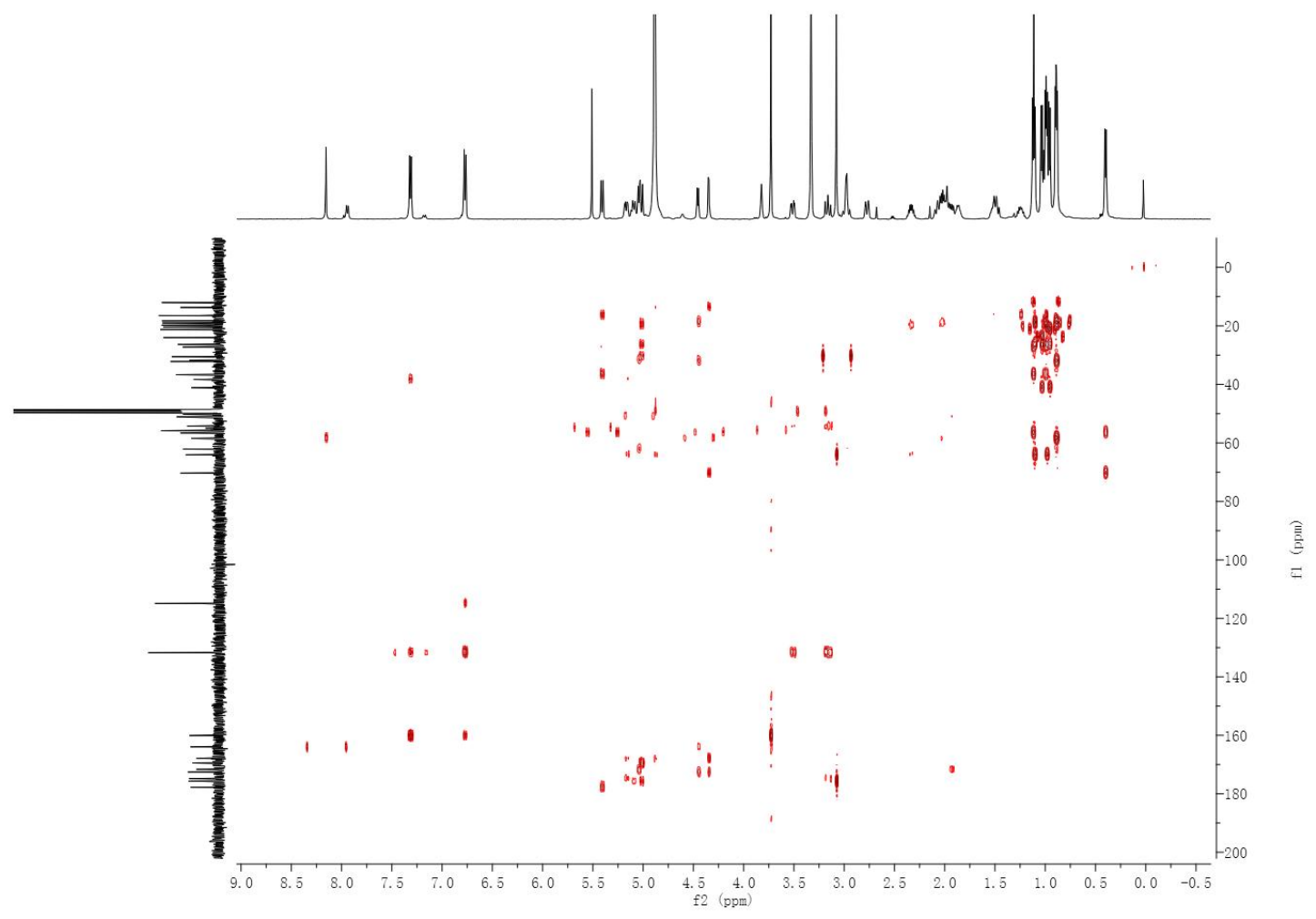

Figure S12. HMBC NMR spectrum of compound 7 in MeOD.

S20 


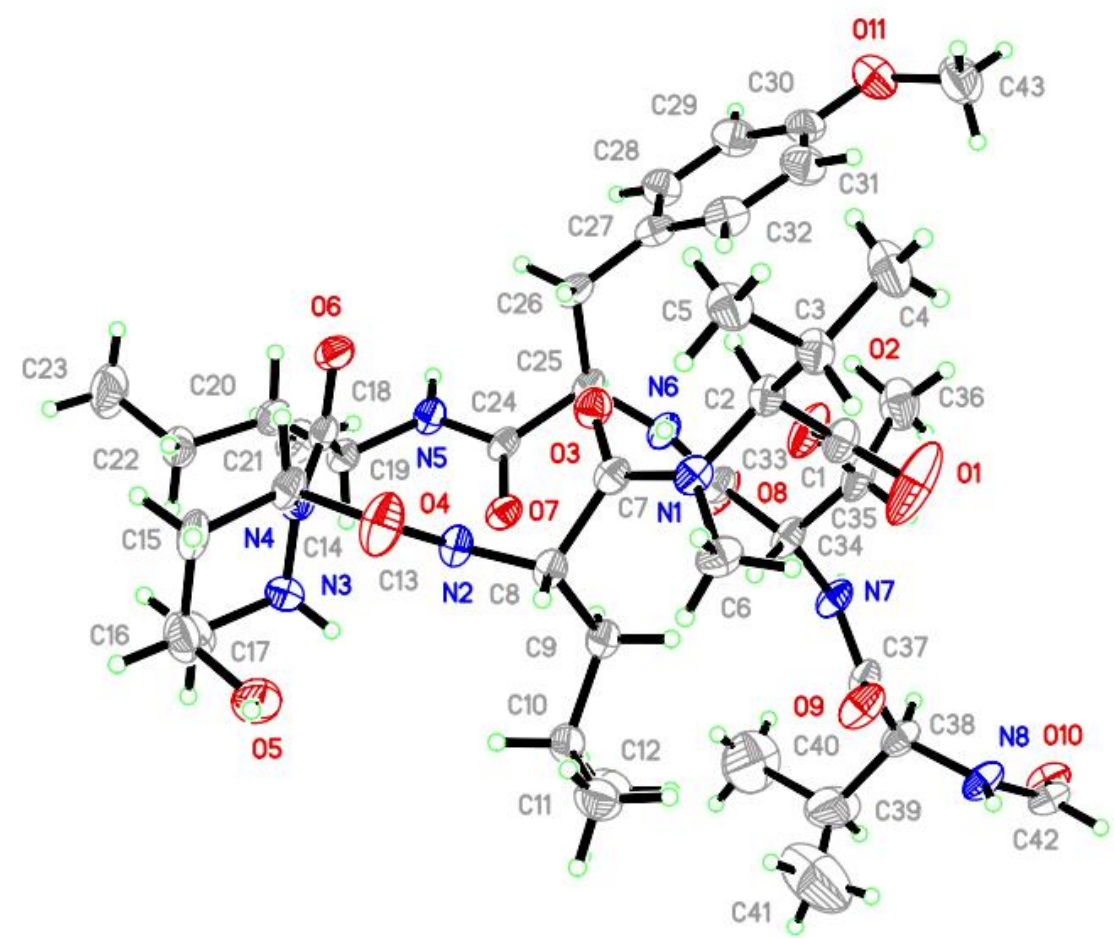

Figure S13. Perspective view of the X-ray structure of 7. 
A

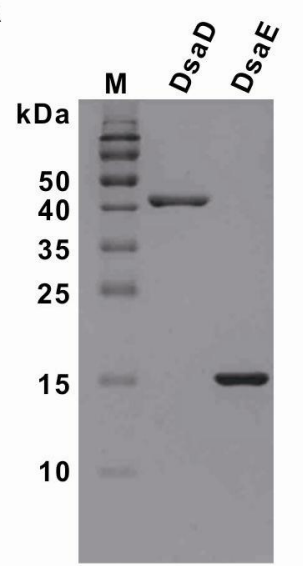

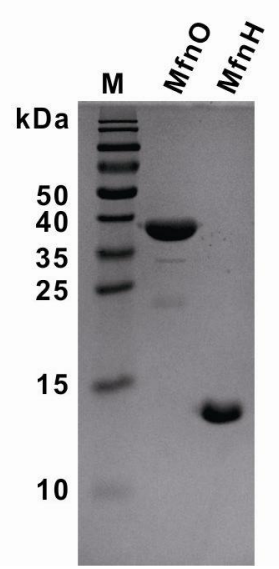

B

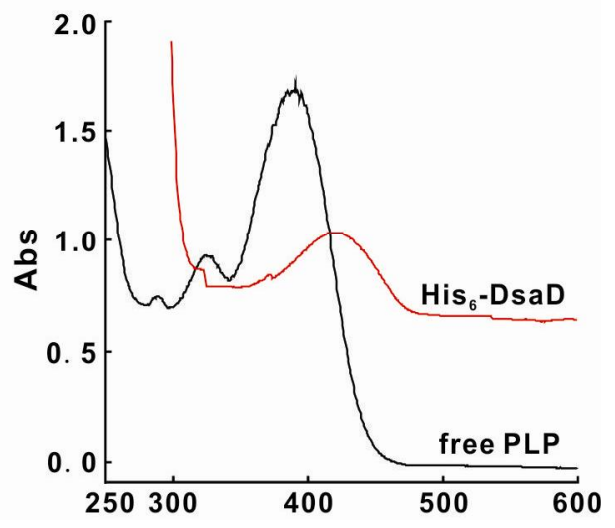

Figure S14. (A) SDS-PAGE analyses of purified $\mathrm{His}_{6}-\mathrm{DsaD}, \mathrm{His}_{6}-\mathrm{DsaE}, \mathrm{His}_{6}-\mathrm{MfnO}$ and $\mathrm{His}_{6}{ }^{-}$ MfnH. (B) UV spectra of $\mathrm{His}_{6}$-DsaD indicate the presence of PLP covalently linked to the catalytic lysine residue of DsaD via a Schiff base linkage. 

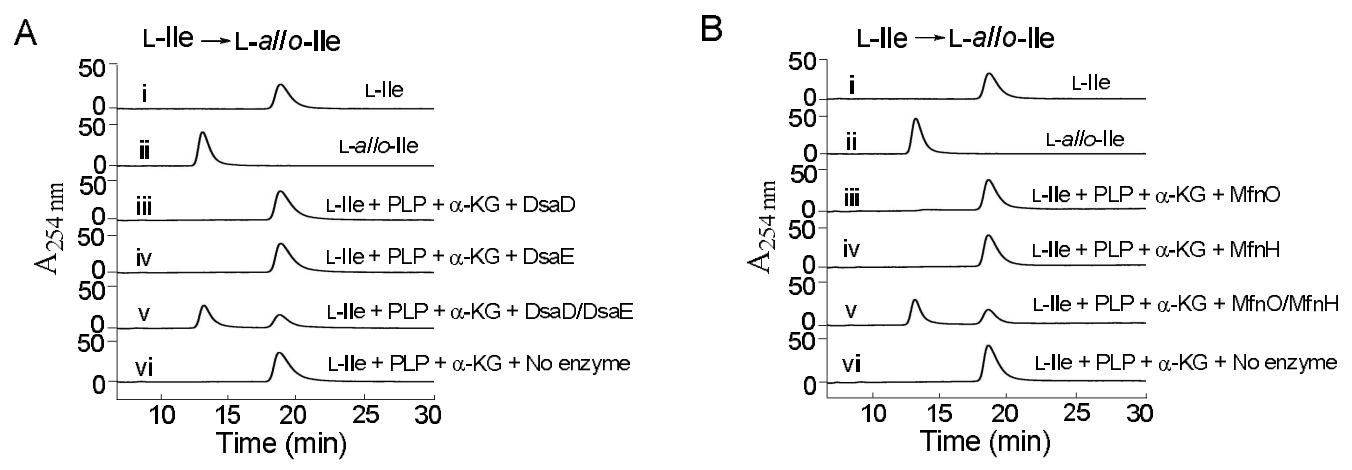

Figure S15. In vitro characterization of DsaD/DsaE and $\mathrm{MfnO} / \mathrm{MfnH}$ in the presence of possible cofactors PLP and $\alpha-K G$. (A) Production of L-allo-lle proceeds only upon co-incubation of L-lle with DsaD and DsaE in the presence of possible cofactors PLP and $\alpha-K G$ (v). Enzyme assays with DsaD alone (iii), DsaE alone (iv), or control lacking DsaD/DsaE (vi) gave only the starting substrate L-lle. (B) Production of L-allo-lle proceeds only upon co-incubation of L-lle with MfnO and $\mathrm{MfnH}$ in the presence of possible cofactors PLP and $\alpha-K G(v)$. Enzyme assays with MfnO alone (iii), $\mathrm{MfnH}$ alone (iv), or control lacking $\mathrm{MfnO} / \mathrm{MfnH}$ (vi) gave only the starting substrate Llle. 

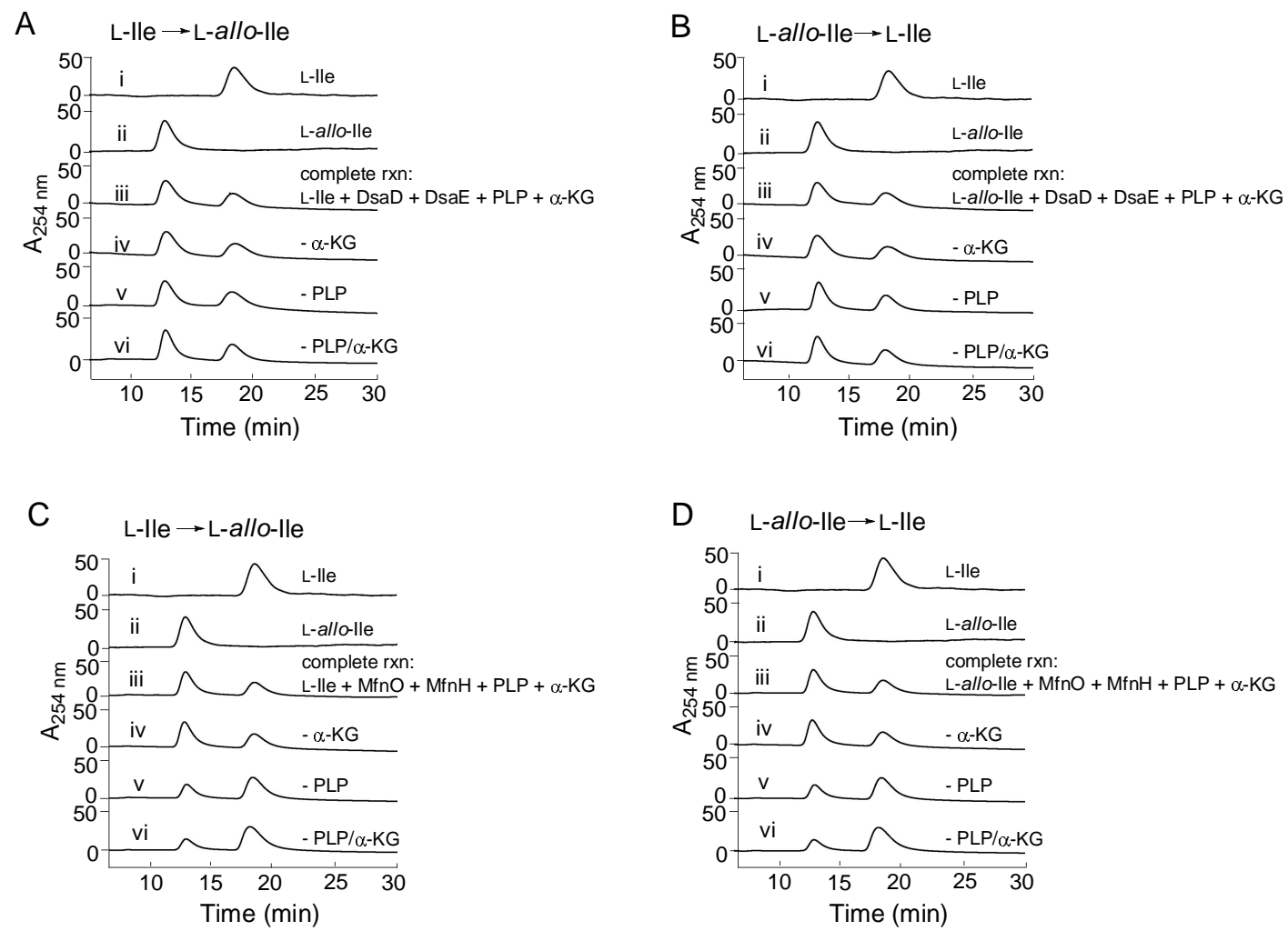

Figure S16. Determination of possible requirement of exogenous PLP and $\alpha$-ketoglutarate ( $\alpha$ $\mathrm{kG}$ ) for the $\mathrm{DsaD} / \mathrm{DsaE}$ or MfnO/MfnH-catalyzed interconversion between L-lle and L-allo-lle. (A) Removal of exogenous PLP (v), $\alpha-K G$ (iv) and PLP/ $\alpha-K G$ (vi) from the complete reaction containing L-lle, DsaD/DsaE, PLP and $\alpha-K G$ has no effect on production of L-allo-lle. (B) Removal of exogenous PLP (v), $\alpha-K G$ (iv) and PLP/ $\alpha-K G$ (vi) from the complete reaction containing L-allo-lle, DsaD/DsaE, PLP and $\alpha-K G$ has no effect on production of L-lle. (C) Removal of exogenous PLP (v), $\alpha-K G$ (iv) and PLP/ $\alpha-K G$ (vi) from the complete reaction containing L-lle, MfnO/MfnH, PLP and $\alpha-K G$ has no effect on production of L-allo-lle. (D) Removal of exogenous PLP (v), $\alpha-K G$ (iv) and PLP/ $\alpha-K G$ (vi) from the complete reaction containing L-allo-lle, MfnO/MfnH, PLP and $\alpha-K G$ has no effect on production of L-lle. 


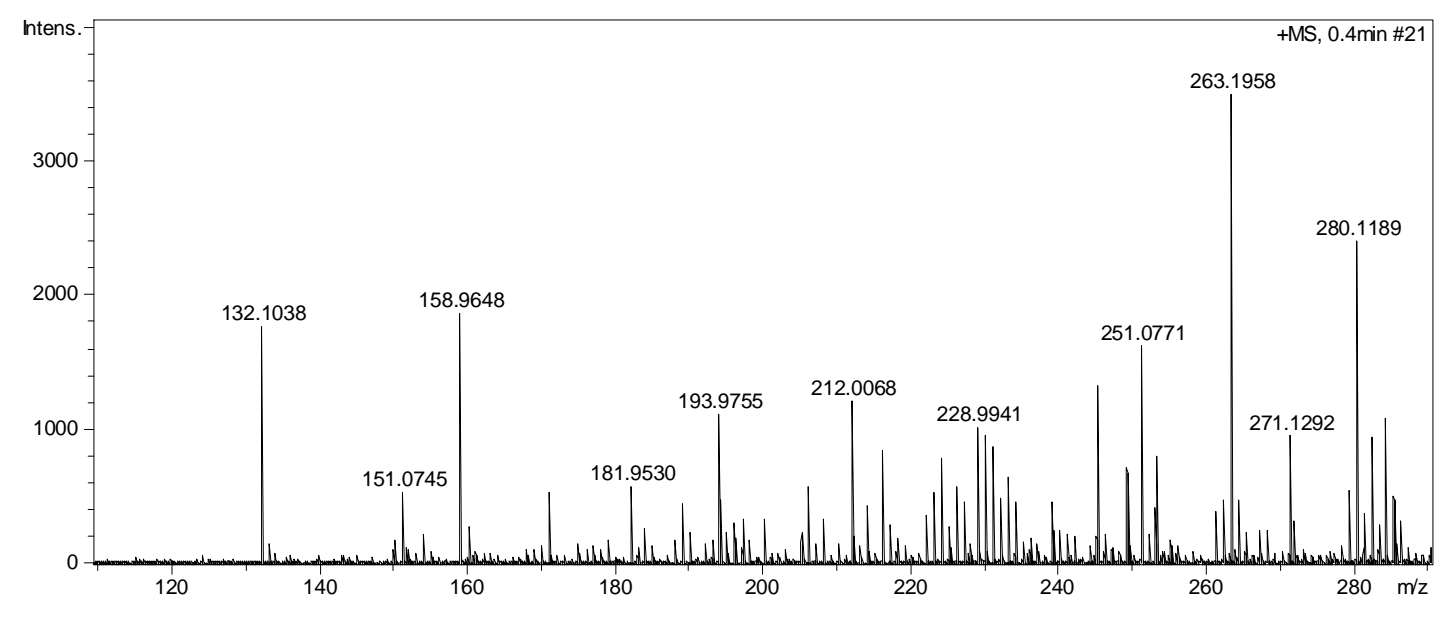

Figure S17. HRESIMS spectrum of the enzymatically generated L-allo-lle. 


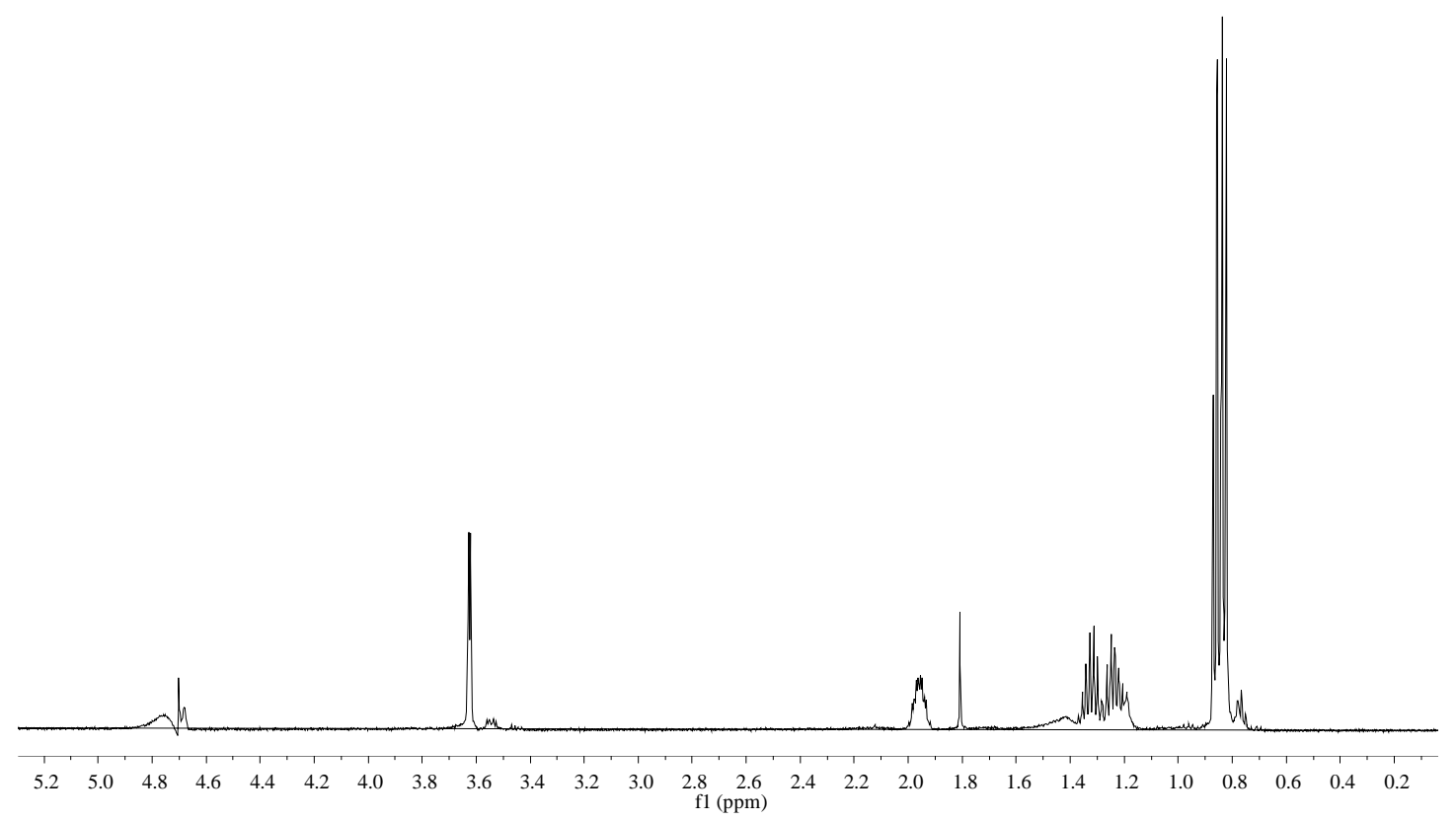

B

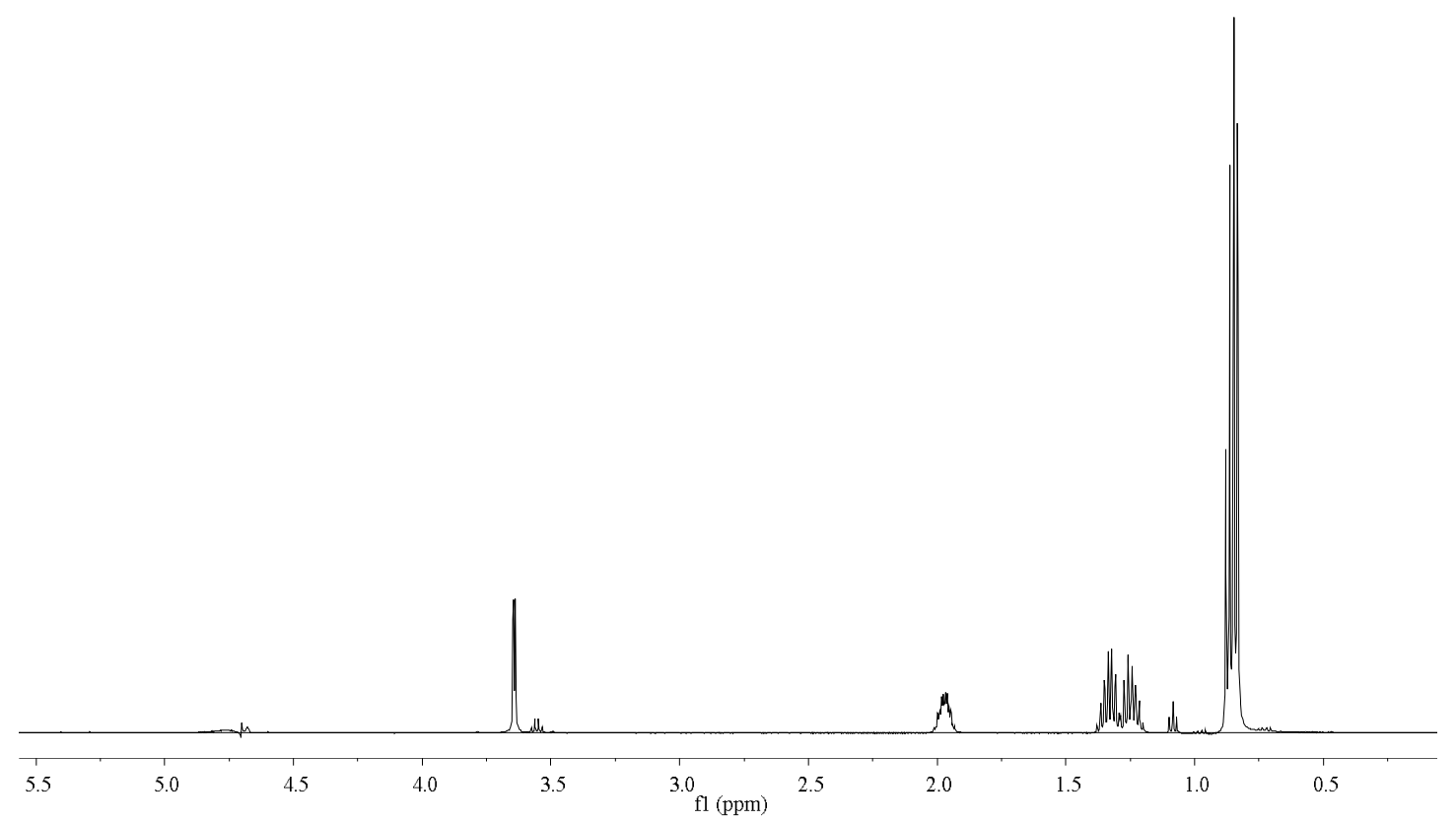

Figure S18. ${ }^{1} \mathrm{H}$ NMR spectra for $(\mathrm{A})$ the enzymatically produced L-allo-lle and $(\mathrm{B})$ the authentic sample of L-allo-lle. 
A
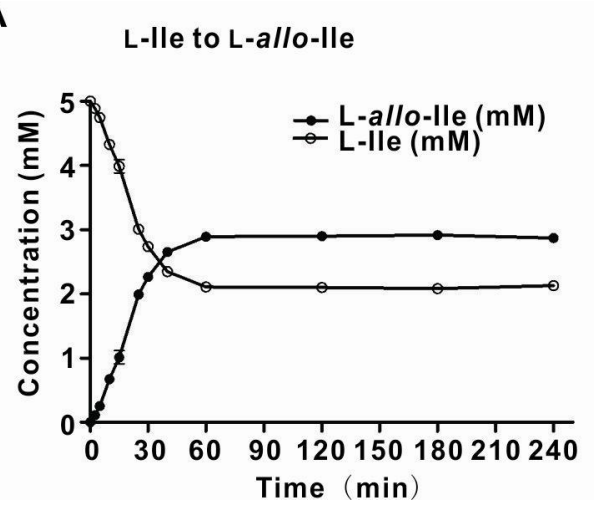

B
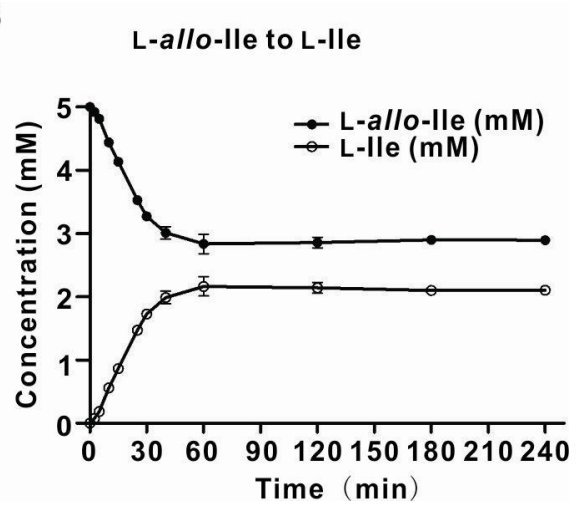

Figure S19. Determination of the equilibrium constant (Keq) for DsaD/DsaE-catalyzed bidirectional reaction with (A) L-lle or (B) L-allo-lle as substrates. Enzyme assays were performed in $50 \mu \mathrm{L}$ sodium phosphate buffer (50 mM, pH8.0) containing $2.5 \mu \mathrm{M} \mathrm{DsaD}, 2.5 \mu \mathrm{M}$ DsaE, $0.1 \mathrm{mM} \mathrm{PLP}$ and $5 \mathrm{mM} \mathrm{L-lle}$ or L-allo-lle as substrate at $30^{\circ} \mathrm{C}$ in triplicate. Reactions were stopped by addition of $150 \mu \mathrm{L}$ of methanol after $2.5,5,10,15,25,30,40,60,120,180,240 \mathrm{~min}$. The Keq was calculated using the equation $K e q=([p r o d u c t] /[s u b s t r a t e])$ at reaction equilibrium. Thus, Keq for the reaction with L-lle as the substrate is 1.37 (Keq $=([\mathrm{L}-$ allo-Ile]/[L-Ile $])=$ $(2.89 / 2.11)=1.37)$. 


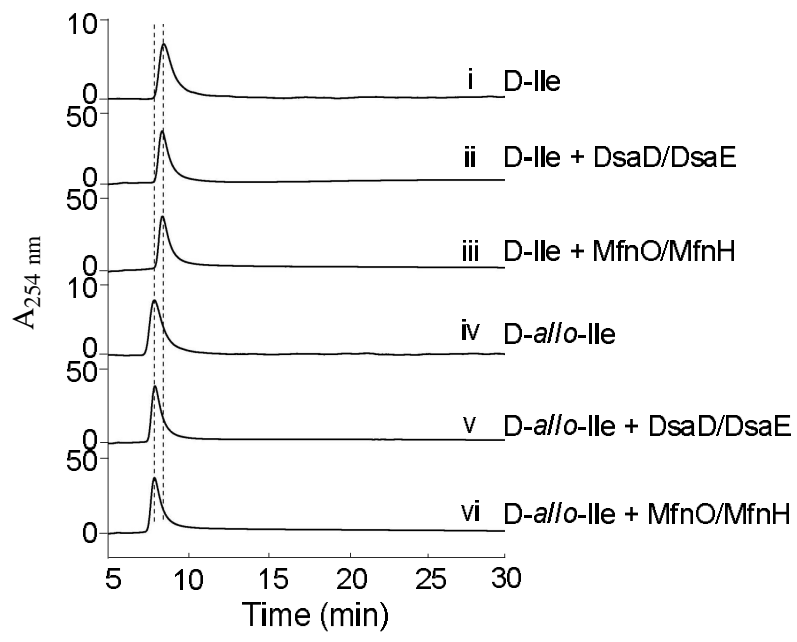

Figure S20. Substrate specificity assays for DsaD/DsaE and MfnO/MfnH. Co-incubation of D-lle with DsaD/DsaE (ii), MfnO/MfnH (iii) yielded no new peaks. Co-incubation of D-allo-lle with DsaD/DsaE (v), MfnO/MfnH (vi) also yielded no new peaks. 

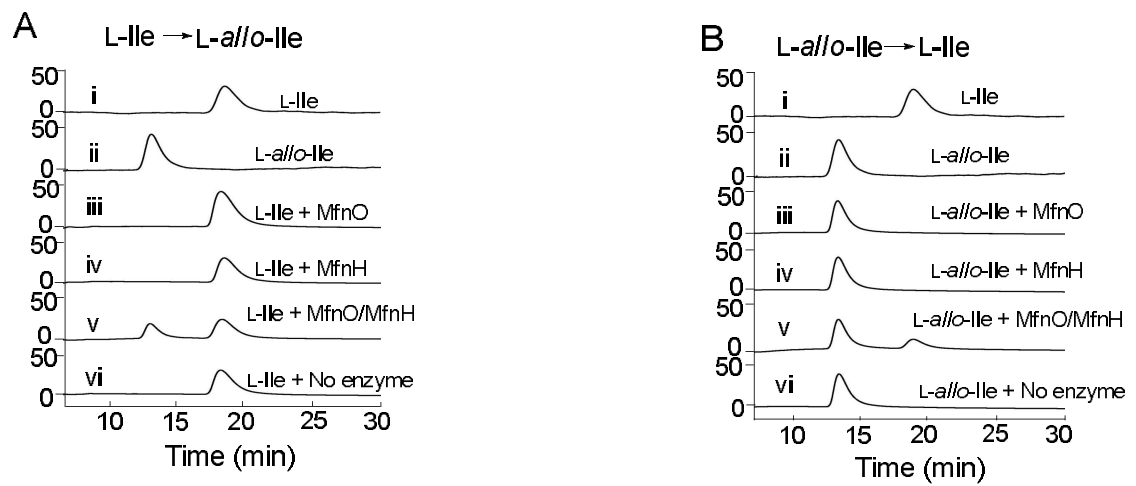

Figure S21. In vitro characterization of $\mathrm{MfnO} / \mathrm{MfnH}$. (A) Production of L-allo-lle proceeds only upon co-incubation of L-lle with $\mathrm{MfnO}$ and $\mathrm{MfnH}$ (v). Enzyme assays with MfnO alone (iii), $\mathrm{MfnH}$ alone (iv), or control lacking MfnO/MfnH (vi) yielded only starting substrate L-lle. (B) Production of L-lle occurs only upon co-incubation of L-allo-lle with $\mathrm{MfnO}$ and $\mathrm{MfnH}$ (v). Enzyme assays with MfnO alone (iii), MfnH alone (iv), or control lacking MfnO/MfnH (vi) afforded only starting substrate L-allo-lle.
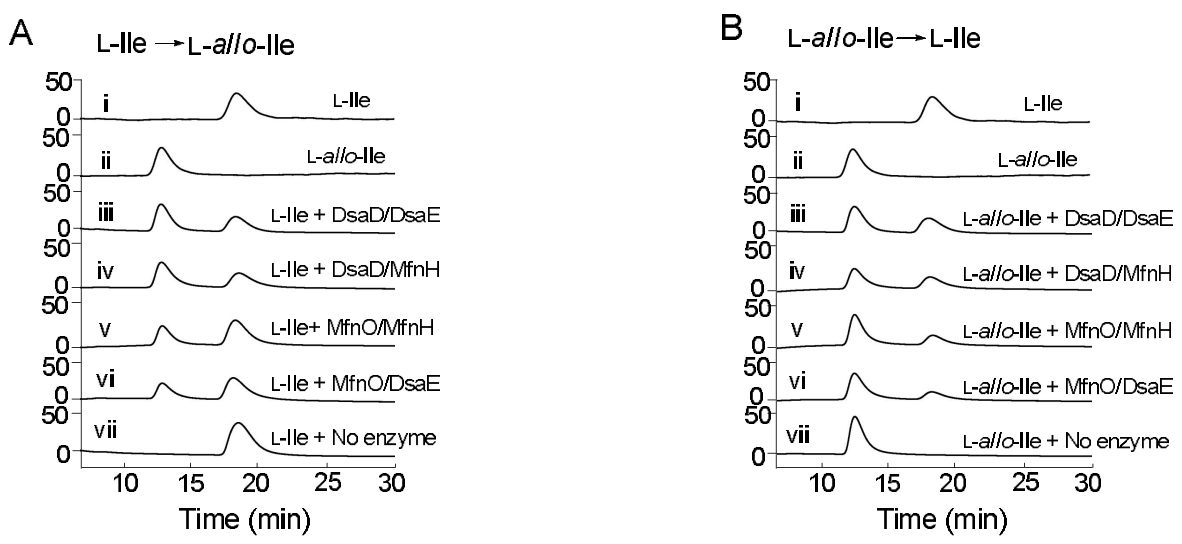

Figure S22. (A) The activity assays of the crossed enzyme pairs DsaD/MfnH and MfnO/DsaE using L-lle as the substrate. L-allo-lle was generated upon co-incubation with L-lle and DsaD/DsaE (iii), DsaD/MfnH (iv), MfnO/MfnH (v) and MfnO/DsaE (vi). Control reaction lacking the enzymes failed to afford of L-allo-lle (vii). (B) The activity assays of the crossed enzyme pairs DsaD/MfnH and MfnO/DsaE using L-allo-lle as the substrate. L-lle was generated upon coincubation of L-allo-lle with DsaD/DsaE (iii), DsaD/MfnH (iv), MfnO/MfnH (v) and MfnO/DsaE (vi). Control reaction lacking the enzymes failed to afford L-lle (vii). 


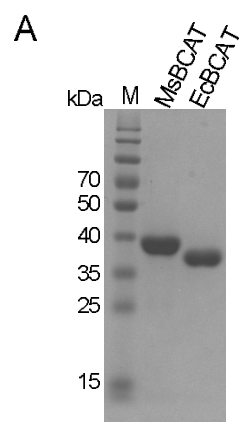

B

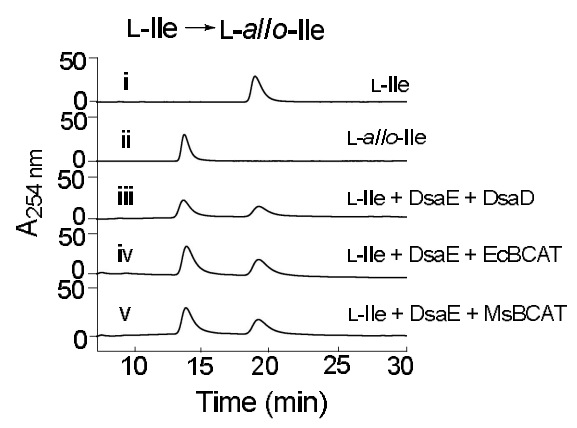

D

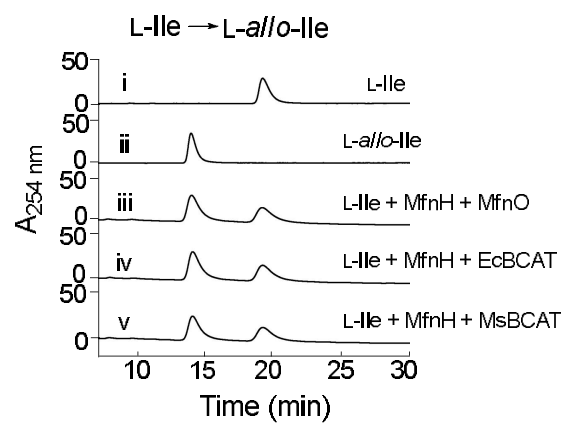

C

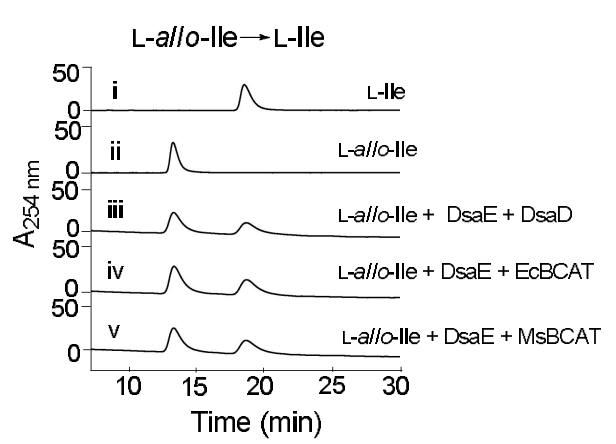

E

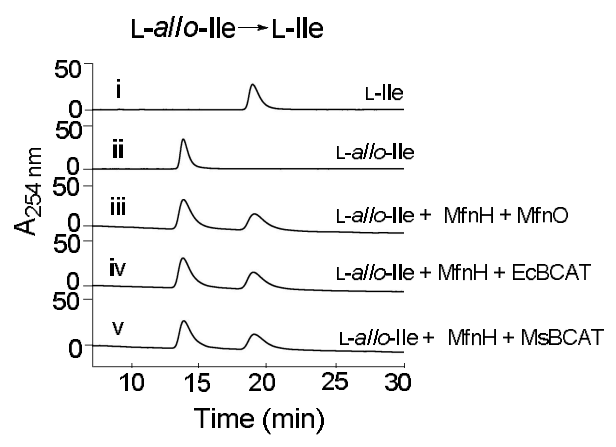

Figurre S23. (A) SDS-PAGE analyses of purified $\mathrm{His}_{6}-\mathrm{EcBCAT}$ and His $\mathrm{H}_{6}$ MsBCAT. M: protein markers. (B - E) Activity assays of EcBCAT and MsBCAT from primary metabolism. Coincubation of EcBCAT or MsBCAT with isomerase DsaE or MfnH using either L-lle or L-allo-lle as substrates under the standard conditions afforded the respective products L-allo-lle or L-Ile. 
A

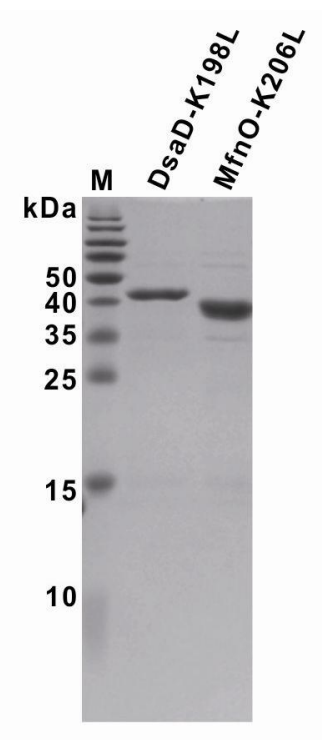

B

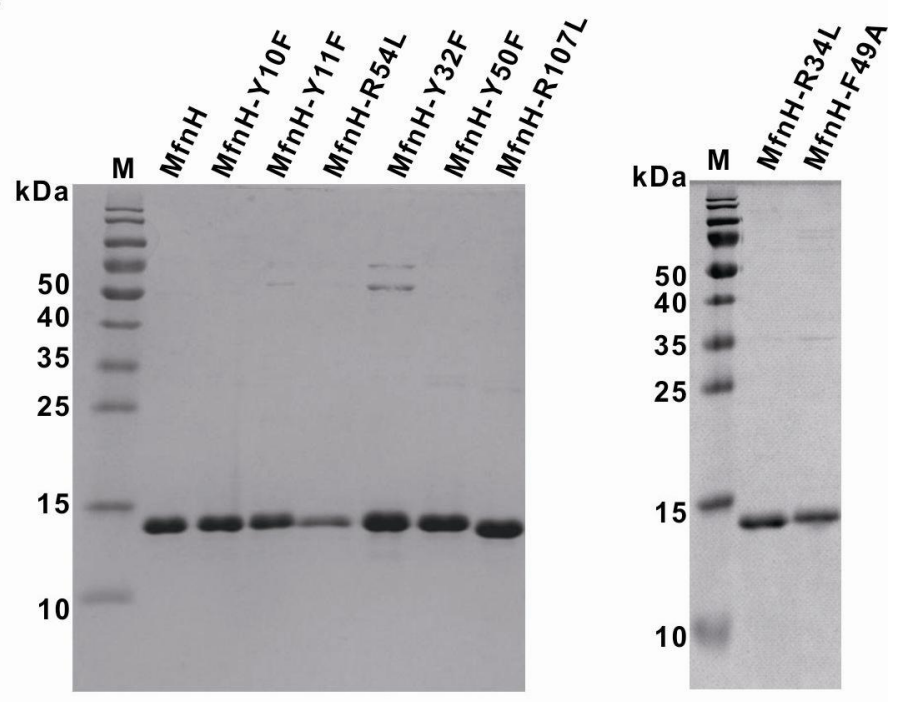

C

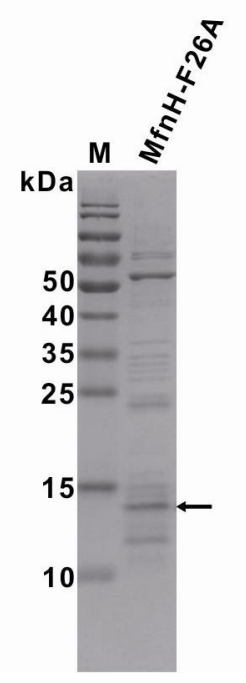

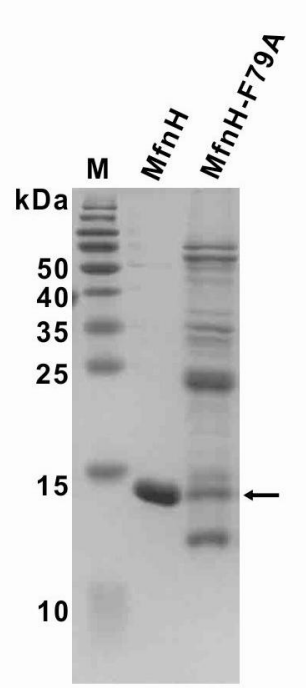

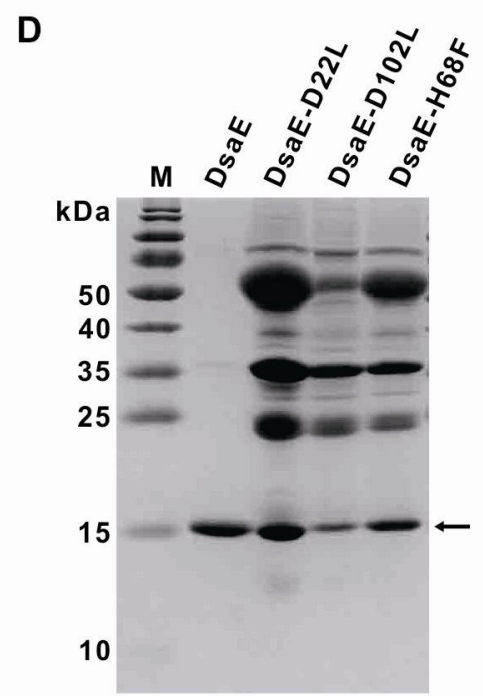

Figure S24. SDS-PAGE analyses of the purified E. coli-derived mutants of DsaD, MfnO, MfnH, and DsaE. (A) SDS-PAGE analyses of the aminotransferase DsaD/MfnO mutants. (B) SDSPAGE analyses of the eight $\mathrm{MfnH}$ mutants purified to homogeneity as soluble proteins. (C) SDS-PAGE analyses of the two partially purified MfnH mutants. (D) SDS-PAGE analyses of the three partially purified DsaE mutants. M: protein marker. 

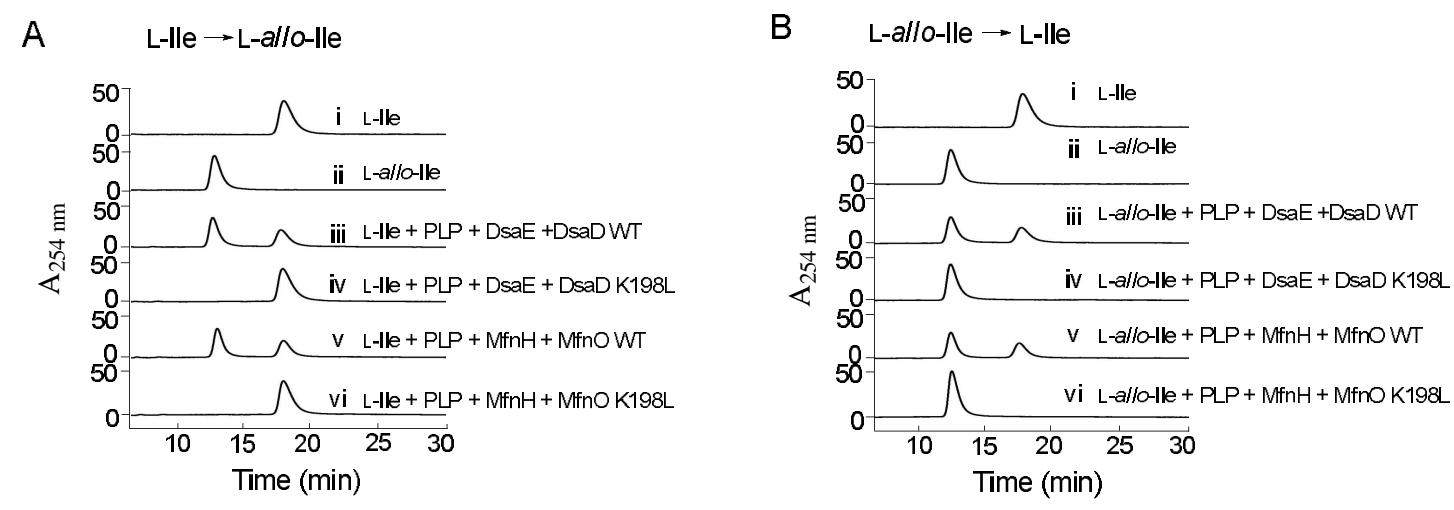

Figure S25. Effect of PLP supplementation upon the DsaD/MfnO mutant enzyme activities. Addition of supplemental PLP to the enzyme reaction mixtures failed to restore DsaD or MfnO enzymatic activities when using (A) L-lle or (B) L-allo-lle as substrates. 
A

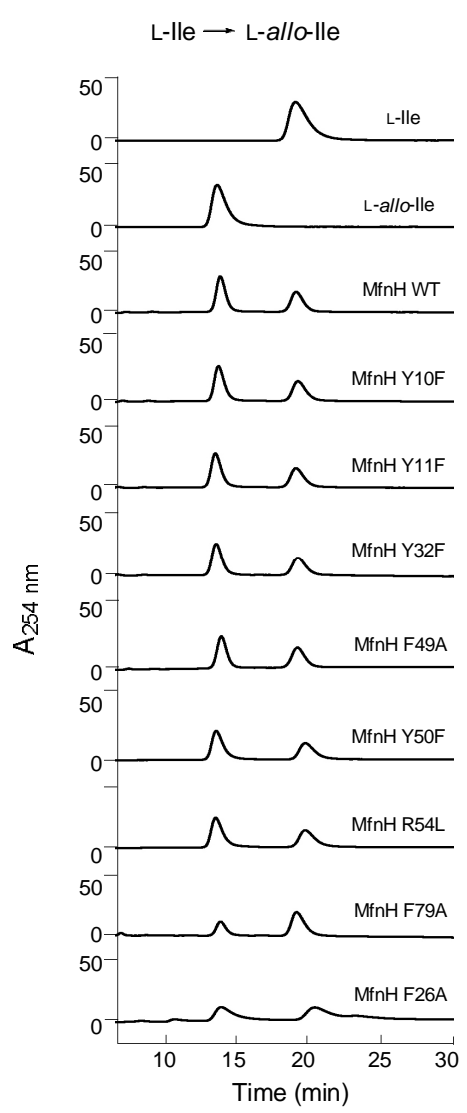

C

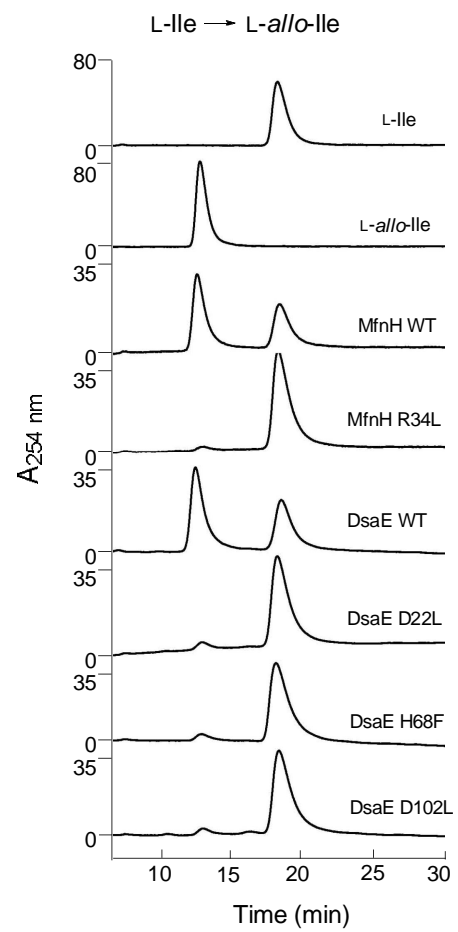

B

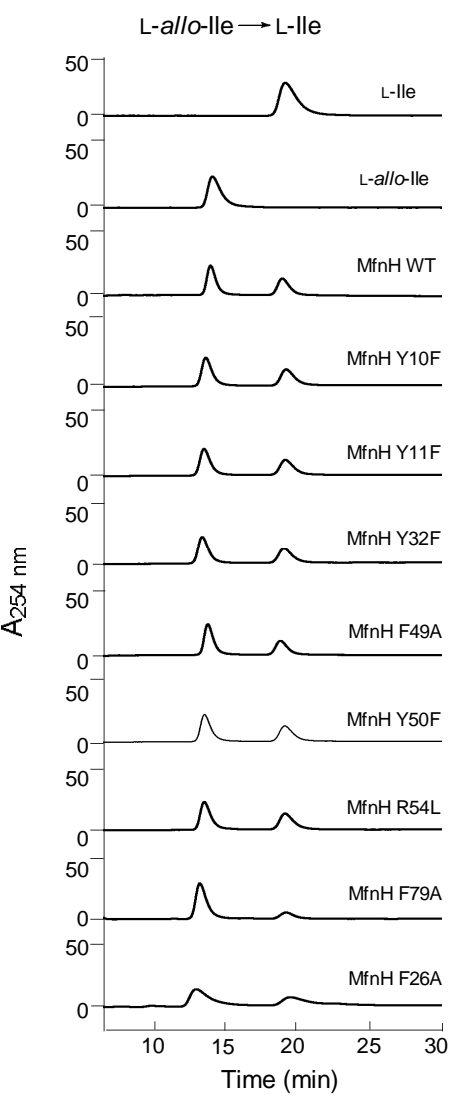

D

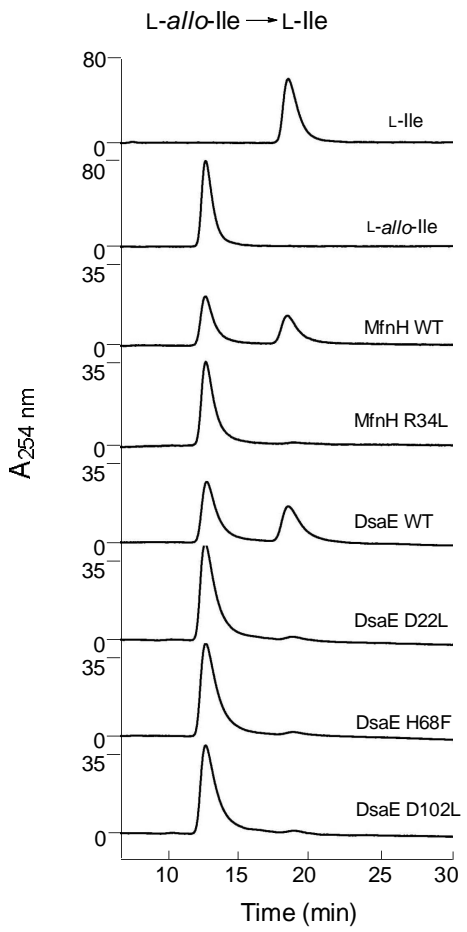


Figure S26. HPLC profiles of the activity assays of the eight MfnH mutants as coupled with MfnO using either (A) L-lle or (B) L-allo-lle as substrates. These eight MfnH mutants displayed no obvious deviations in enzymatic activity relative to wild-type enzyme. Also shown are HPLC profiles of the activity assays of the four MfnH/DsaE mutants as coupled with MfnO or DsaD using (C) L-Ile or (D) L-allo-lle as substrates. These four MfnH/DsaE mutants displayed sharply diminished enzymatic activity corresponding to $~ 8 \%$ of that displayed by wild-type MfnH/DsaE. 


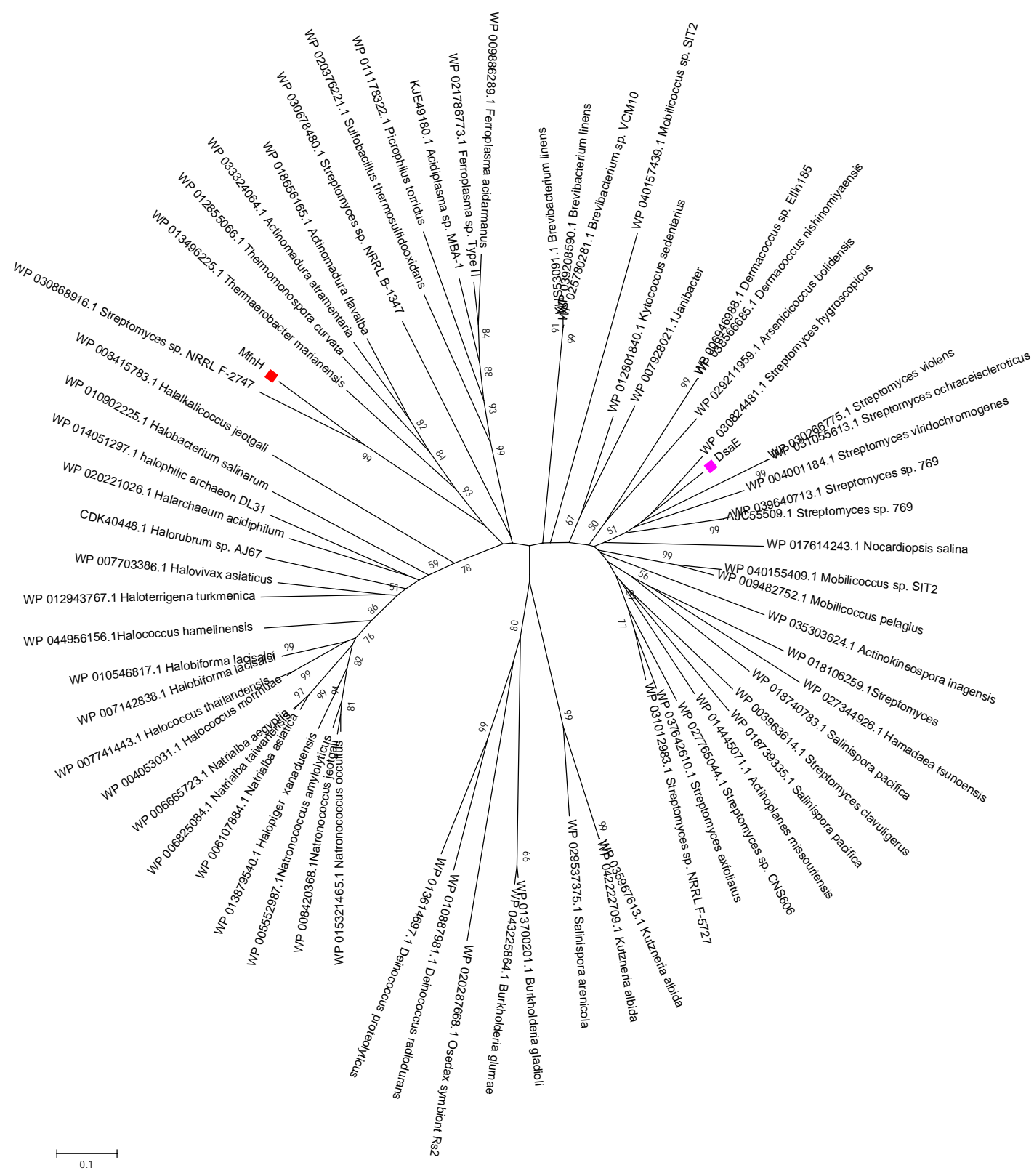

Figure S27. Phylogenetic tree of DsaE/MfnH with their homologues. The amino acid sequences were aligned using ClustalX (2.1) and the phylogenetic tree was generated using Molecular Evolutionary Genetics Analysis (MEGA) 6.0. 